\title{
The Strominger-Yau-Zaslow conjecture: From torus fibrations to degenerations.
}

\author{
Mark Gross
}

\begin{abstract}
We trace progress and thinking about the Strominger-Yau-Zaslow conjecture since its introduction in 1996. In particular, we aim to explain how the conjecture led to the algebro-geometric program developed by myself and Siebert, whose objective is to explain mirror symmetry by studying degenerations of Calabi-Yau manifolds. We end by outlining how tropical curves arise in the mirror symmetry story.
\end{abstract}

\section{Introduction.}

Up to the summer of 1996, there had been a number of spectacular successes in mirror symmetry. After the pioneering initial work of Candelas, de la Ossa, Greene and Parkes 8 which calculated the instanton predictions for the quintic three-fold, Batyrev [2 gave a powerful mirror symmetry construction for CalabiYau hypersurfaces in toric varieties, later generalized by Batyrev and Borisov $\mathbf{3}$ to complete intersections in toric varieties. Kontsevich 37 introduced his notion of stable maps of curves and studied their moduli, setting the stage for a new flowering of enumerative geometry. Eventually, this led to the mathematical calculation of Gromov-Witten invariants for the quintic in varying forms, 13, 43, 6, 12. In between, a great deal of the structure of mirror symmetry was elucidated by many researchers in both string theory and algebraic geometry.

On the other hand, at that time I had been primarily interested in the geometry of Calabi-Yau manifolds. Many of the results about mirror symmetry seemed to rely primarily on information about the ambient toric varieties; in particular, Givental's work 13 and succeeding work by Lian, Liu and Yau [3, Bertram 6 and Gathmann 12 always performed calculations on the moduli space of stable maps into $\mathbb{P}^{4}$ rather than the quintic, and in the Batyrev-Borisov type constructions, while there was a clear combinatorial relationship between the ambient toric varieties, there was no apparent geometric relationship between the Calabi-Yaus themselves. As a result, I tended to avoid thinking about mirror symmetry precisely because of this lack of geometric understanding.

2000 Mathematics Subject Classification. 14J32.

This work was partially supported by NSF grant 0505325 .

(C)0000 (copyright holder) 
This changed dramatically in June of 1996, when Strominger, Yau and Zaslow 56 released their paper "Mirror Symmetry is T-duality." They made a remarkable proposal, based on recent ideas in string theory, that for the first time gave a geometric interpretation for mirror symmetry.

Let me summarize, very roughly, the physical argument here. Developments in string theory in the mid-1990s had introduced the notion of Dirichlet branes, or $D$-branes. These are submanifolds of space-time, with some additional data, which should serve as a boundary condition for open strings, i.e. we allow open strings to propagate with their endpoints constrained to lie on a $D$-brane. Remembering that space-time, according to string theory, looks like $\mathbb{R}^{1,3} \times X$, where $\mathbb{R}^{1,3}$ is ordinary space-time and $X$ is a Calabi-Yau three-fold, we can split a $D$-brane into a product of a submanifold of $\mathbb{R}^{1,3}$ and one on $X$. It turned out, simplifying a great deal, that there were two particular types of submanifolds on $X$ of interest: holomorphic Dbranes, i.e. holomorphic submanifolds with a holomorphic line bundle, and special Lagrangian $D$-branes, which are special Lagrangian submanifolds with flat $U(1)$ bundle:

Definition 0.1. Let $X$ be an $n$-dimensional Calabi-Yau manifold with $\omega$ the Kähler form of a Ricci-flat metric on $X$ and $\Omega$ a nowhere vanishing holomorphic $n$-form. Then a submanifold $M \subseteq X$ is special Lagrangian if it is Lagrangian, i.e. $\operatorname{dim}_{\mathbb{R}} M=\operatorname{dim}_{\mathbb{C}} X$ and $\left.\omega\right|_{M}=0$, and in addition $\left.\operatorname{Im} \Omega\right|_{M}=0$.

The origins of mirror symmetry in physics suggest that if $X$ and $\check{X}$ are a mirror pair of Calabi-Yau manifolds, then string theory on a compactification of space-time using $X$ should be the same as that using $\check{X}$, but with certain data interchanged. In the case of $D$-branes, the suggestion is that the moduli space of holomorphic $D$-branes on $X$ should be isomorphic to the moduli space of special Lagrangian $D$-branes on $\check{X}$. Now $X$ itself is the moduli space of points on $X$. So each point on $X$ should correspond to a pair $(M, \nabla)$, where $M \subseteq \check{X}$ is a special Lagrangian submanifold and $\nabla$ is a flat connection on $M$.

A theorem of McLean [45 tells us that the tangent space to the moduli space of special Lagrangian deformations of a special Lagrangian submanifold $M \subseteq \check{X}$ is $H^{1}(M, \mathbb{R})$. Of course, the moduli space of flat $U(1)$-connections modulo gauge equivalence on $M$ is the torus $H^{1}(M, \mathbb{R}) / H^{1}(M, \mathbb{Z})$. In order for this moduli space to be of the correct dimension, we need $\operatorname{dim} H^{1}(M, \mathbb{R})=n$, the complex dimension of $X$. This suggests that $X$ consists of a family of tori which are dual to a family of special Lagrangian tori on $\check{X}$. An elaboration of this argument yields the following conjecture:

Conjecture 0.2. The Strominger-Yau-Zaslow conjecture. If $X$ and $\check{X}$ are a mirror pair of Calabi-Yau n-folds, then there exists fibrations $f: X \rightarrow B$ and $\check{f}: \check{X} \rightarrow B$ whose fibres are special Lagrangian, with general fibre an $n$-torus. Furthermore, these fibrations are dual, in the sense that canonically $X_{b}=H^{1}\left(\check{X}_{b}, \mathbb{R} / \mathbb{Z}\right)$ and $\check{X}_{b}=H^{1}\left(X_{b}, \mathbb{R} / \mathbb{Z}\right)$ whenever $X_{b}$ and $\check{X}_{b}$ are non-singular tori.

I will clarify this statement as we review the work of the past ten years; however, as I have stated this conjecture, it is likely to be false. On the other hand, there are weaker versions of the conjecture which probably are true. Even better, these weaker statements are probably within reach of modern-day technology (with a lot of hard work). Nevertheless, there has been a lot of good progress on precise versions of the above conjecture at the topological and symplectic level. In addition, 
the conjecture has been successful at explaining many features of mirror symmetry, some of which are still heuristic and some of which are rigorous. It is my belief that a final satisfactory understanding of mirror symmetry will flow from the SYZ conjecture, even if results do not take the form initially suggested by it.

My main goal here is to explain the journey taken over the last ten years. I want to focus on explaining the evolution and development of the ideas, rather than focus on precise statements. Except in the first few sections, I will give few precise statements.

In those first sections, I will clarify the above statement of the conjecture, and show how it gives a satisfactory explanation of mirror symmetry in the so-called semi-flat case, i.e. the case when the metric along the special Lagrangian fibres is flat. This leads naturally to a discussion of affine manifolds, metrics on them, and the Legendre transform. These now appear to be the key structures underlying mirror symmetry.

We next take a look at the case when singular fibres appear. In this case we need to abandon the precise form of duality we developed in the semi-flat case and restrict our attention to topological duality. In the realm of purely topological duality, the SYZ conjecture has been entirely successful at explaining topological features of mirror symmetry for a large range of Calabi-Yau manifolds, including those produced by the Batyrev-Borisov construction for complete intersections in toric varieties.

Moving on, we take a look at Dominic Joyce's arguments demonstrating the problems with the strong form of the SYZ conjecture stated above. This forces us to recast the SYZ conjecture as a limiting statement. Mirror symmetry is always about the behaviour of Calabi-Yau manifolds near maximally unipotent degenerations. A limiting form of the SYZ conjecture suggests that one can find special Lagrangian tori on Calabi-Yau manifolds near a maximally unipotent degeneration, and as we approach the limit point in complex moduli space, we expect to see a larger portion of the Calabi-Yau manifold filled out by special Lagrangian tori. Unlike the original SYZ conjecture, though still difficult, this one looks likely to be accessible by current techniques.

This form of the conjecture then motivates a new round of questions. In this limiting picture, we expect the base $B$ of the hypothetical special Lagrangian fibration to be the so-called Gromov-Hausdorff limit of a sequence of Calabi-Yau manifolds approaching the maximally unipotent degeneration. Gromov-Hausdorff convergence is a metric space concept, while maximally unipotent degeneration is an algebro-geometric, Hodge-theoretic concept. How do these two concepts relate? In the summer of 2000, Kontsevich suggested that the Gromov-Hausdorff limit will be, roughly, the dual intersection complex of the algebro-geometric degeneration, at least on a topological level. We explore this idea in $\S 6$.

On the other hand, how does this help us with mirror symmetry? Parallel to these developments on limiting forms of the SYZ conjecture, my coauthor Bernd Siebert had been studying degenerations of Calabi-Yau manifolds using logarithmic geometry with Stefan Schröer. Siebert noticed that mirror symmetry seemed to coincide with a combinatorial exchange of logarithmic data on the one side and polarizations on the other. Together, we realised that this approach to mirror symmetry meshed well with the limiting picture predicted by SYZ. Synthesizing these two approaches, we discovered an algebro-geometric version of the SYZ approach, 
which I will describe here. The basic idea is to forget about special Lagrangian fibrations, and only keep track of the base of the fibration, which is an affine manifold. We show how polyhedral decompositions of affine manifolds give rise to degenerate Calabi-Yau varieties, and conversely how certain sorts of degenerations of CalabiYau varieties, which we call toric degenerations, give rise to affine manifolds as their dual intersection complex. Mirror symmetry is again explained by a discrete version of the Legendre transform much as in the semi-flat case.

We end with a discussion of the connection of tropical curves with this approach. The use of tropical curves in curve counting in two-dimensional toric varieties has been pioneered in work of Mihkalkin [46; Nishinou and Siebert 48, generalized this work to higher dimensions using an approach directly inspired by the approach I discuss here. On the other hand, tropical curves have not yet been used for counting curves in Calabi-Yau manifolds, so I will end the paper by discussing how tropical curves arise naturally in our picture.

I would like to thank the organizers of the Seattle conference for running an excellent conference, and my coauthors Pelham Wilson and Bernd Siebert on SYZ related results; much of the work mentioned here came out of work with them.

\section{First a topological observation.}

Before doing anything else, let's ask a very basic question: why should dualizing torus fibrations interchange Hodge numbers of Calabi-Yau threefolds? If you haven't seen this, it's the first thing one should look at as it is particularly easy to see, if we make a few assumptions. Suppose we are given a pair of Calabi-Yau threefolds $X$ and $\check{X}$ with fibrations $f: X \rightarrow B, \check{f}: \check{X} \rightarrow B$ with the property that there is a dense open set $B_{0} \subseteq B$ such that $f_{0}: f^{-1}\left(B_{0}\right) \rightarrow B_{0}$ and $\check{f}_{0}: \check{f}^{-1}\left(B_{0}\right) \rightarrow B_{0}$ are torus fibre bundles. So all the singular fibres of $f$ and $\check{f}$ lie over $\Gamma:=B \backslash B_{0}$. (Note that unless $\chi(X)=0$, there must be some singular fibres.) Finally, assume $f_{0}$ and $\check{f}_{0}$ are dual torus fibrations, i.e. $f_{0}$ can be identified with the torus fibration $R^{1} \check{f}_{0 *}(\mathbb{R} / \mathbb{Z}) \rightarrow B_{0}$ and $\check{f}_{0}$ can be identified with the torus fibration $R^{1} f_{0 *}(\mathbb{R} / \mathbb{Z}) \rightarrow B_{0}$. (This is a slight abuse of notation: by $R^{1} \check{f}_{0 *}(\mathbb{R} / \mathbb{Z})$ we really mean the torus bundle obtained by taking the vector bundle associated to the local system $R^{1} \breve{f}_{0 *} \mathbb{R}$ and dividing out by the family of lattices $R^{1} \check{f}_{0 *} \mathbb{Z}$.)

If $V / \Lambda$ is a single torus with $V$ an $n$-dimensional vector space and $\Lambda$ a lattice in $V$, then $H^{p}(V / \Lambda, \mathbb{R}) \cong \Lambda^{p} V^{\vee}$, while the dual torus, $V^{\vee} / \Lambda^{\vee}$, has $H^{p}\left(V^{\vee} / \Lambda^{\vee}, \mathbb{R}\right) \cong$ $\bigwedge^{p} V$. If we choose an isomorphism $\Lambda^{n} V \cong \mathbb{R}$, then we get an isomorphism $H^{p}(V / \Lambda, \mathbb{R}) \cong H^{n-p}\left(V^{\vee} / \Lambda^{\vee}, \mathbb{R}\right)$. Similarly, in the relative setting for $f_{0}$ and $\check{f}_{0}$, if we have an isomorphism $R^{3} f_{0 *} \mathbb{R} \cong \mathbb{R}$, we obtain isomorphisms

$$
R^{p} f_{0 *} \mathbb{R} \cong R^{3-p} \check{f}_{0 *} \mathbb{R} .
$$

We now make a simplifying assumption. Let $i: B_{0} \hookrightarrow B$ be the inclusion. We will say $f$ is $\mathbb{R}$-simple if

$$
i_{*} R^{p} f_{0 *} \mathbb{R} \cong R^{p} f_{*} \mathbb{R}
$$

for all $p$. (We can in general replace $\mathbb{R}$ by any abelian group $G$, and then we say $f$ is $G$-simple.) Of course, not all torus fibrations are $\mathbb{R}$-simple, but it turns out that the most interesting ones which occur in the topological form of SYZ are. So let's assume $f$ and $\check{f}$ are $\mathbb{R}$-simple. With this assumption, we obtain isomorphisms

$$
R^{p} f_{*} \mathbb{R} \cong R^{3-p} \check{f}_{*} \mathbb{R} .
$$


We can now use this to study the Leray spectral sequence for $f$ and $\check{f}$.

Let's make an additional assumption that $X$ and $\check{X}$ are simply connected. So in particular $B$ is simply connected. Let's assume $B$ is a three-manifold. So we have the $E_{2}$ terms in the Leray spectral sequence for $f$ :

$\begin{array}{cccc}\mathbb{R} & 0 & 0 & \mathbb{R} \\ H^{0}\left(B, R^{2} f_{*} \mathbb{R}\right) & H^{1}\left(B, R^{2} f_{*} \mathbb{R}\right) & H^{2}\left(B, R^{2} f_{*} \mathbb{R}\right) & H^{3}\left(B, R^{2} f_{*} \mathbb{R}\right) \\ H^{0}\left(B, R^{1} f_{*} \mathbb{R}\right) & H^{1}\left(B, R^{1} f_{*} \mathbb{R}\right) & H^{2}\left(B, R^{1} f_{*} \mathbb{R}\right) & H^{3}\left(B, R^{1} f_{*} \mathbb{R}\right) \\ \mathbb{R} & 0 & 0 & \mathbb{R}\end{array}$

Since $X$ is simply connected, $H^{1}(X, \mathbb{R})=H^{5}(X, \mathbb{R})=0$, from which we conclude that $H^{0}\left(B, R^{1} f_{*} \mathbb{R}\right)=H^{3}\left(B, R^{2} f_{*} \mathbb{R}\right)=0$. The same argument works for $\check{f}$, and then (11) gives $H^{0}\left(B, R^{2} f_{*} \mathbb{R}\right) \cong H^{0}\left(B, R^{1} \check{f}_{*} \mathbb{R}\right)=0$ and similarly $H^{3}\left(B, R^{1} f_{*} \mathbb{R}\right)=$ 0 . Finally, consider the possible non-zero maps for the spectral sequence:

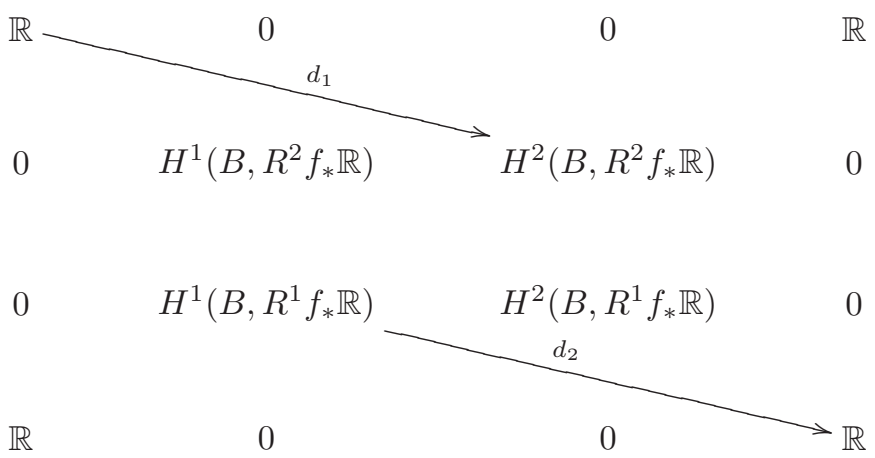

We need one further assumption, which is again natural given the duality relationship of $f_{0}$ and $\check{f}_{0}$ : both $f$ and $\check{f}$ possess sections. (Actually, working over $\mathbb{R}$, we just need the existence of cohomology classes on $X$ and $\bar{X}$ which evaluate to something non-zero on a fibre of $f$ or $\check{f}$.) A section intersects each fibre non-trivially, and hence gives a section of $R^{3} f_{*} \mathbb{R}$. Since such a section also represents a cohomology class on $X, d_{1}$ must be the zero map. Similarly, a fibre of $f$ cannot be homologically trivial because it intersects the section non-trivially, and thus the map $d_{2}$ must be zero. So the spectral sequence degenerates at $E_{2}$. In particular, we get $\mathbb{R}^{h^{1,1}} \cong H^{2}(X, \mathbb{R}) \cong H^{1}\left(B, R^{1} f_{*} \mathbb{R}\right) \cong H^{1}\left(B, R^{2} \check{f}_{*} \mathbb{R}\right)$ and $\mathbb{R}^{h^{2,2}} \cong H^{4}(X, \mathbb{R}) \cong H^{2}\left(B, R^{2} f_{*} \mathbb{R}\right) \cong H^{2}\left(B, R^{1} \check{f}_{*} \mathbb{R}\right)$, where $h^{p, q}$ are the Hodge numbers of $X$. Thus the third Betti number of $\check{X}$ is $2+h^{1,1}+h^{2,2}=2\left(1+h^{1,1}\right)$, so we see $h^{1,1}(X)=h^{1,2}(\check{X})$ and $h^{1,2}(X)=h^{1,1}(\check{X})$.

So modulo some assumptions which would of course eventually have to be justified, it is clear, at least in the three-dimensional case, why the Hodge numbers are interchanged by duality.

More generally, in any dimension, one might hope that $\mathbb{R}$-simplicity implies $\operatorname{dim}_{\mathbb{R}} H^{p}\left(B, R^{q} f_{*} \mathbb{R}\right)=h^{p, q}$, and then a more general exchange of Hodge numbers becomes clear. See Theorem 8.10 for a related result.

REMARK 1.1. This argument can be refined over $\mathbb{Z}$ to make new predictions about the behaviour of integral cohomology under mirror symmetry. In [16. Theorem 3.10, it was shown, again in the three-dimensional case, that if $f$ and $\check{f}$ are 
$\mathbb{Z}$-simple and $\mathbb{Q} / \mathbb{Z}$-simple, $f$ and $\check{f}$ have sections, and $H^{1}(X, \mathbb{Z})=0$, then

$$
\begin{aligned}
H^{\text {even }}(X, \mathbb{Z}[1 / 2]) & \cong H^{\text {odd }}(\check{X}, \mathbb{Z}[1 / 2]) \\
H^{\text {odd }}(X, \mathbb{Z}[1 / 2]) & \cong H^{\text {even }}(\check{X}, \mathbb{Z}[1 / 2])
\end{aligned}
$$

There are problems in the argument with two-torsion, but it is likely the above isomorphisms hold over $\mathbb{Z}$. See 4 for evidence for this latter conjecture.

Enough speculation. Now let's get serious about the structure of special Lagrangian fibrations.

\section{Moduli of special Lagrangian submanifolds}

The first step in really understanding the SYZ conjecture is to examine the structures which arise on the base of a special Lagrangian fibration. These structures arise from McLean's theorem on the moduli space of special Lagrangian submanifolds 45, and these structures and their relationships were explained by Hitchin in 30. We outline some of these ideas here. McLean's theorem says that the moduli space of deformations of a compact special Lagrangian submanifold of a compact Calabi-Yau manifold $X$ is unobstructed, with tangent space at $M \subseteq X$ special Lagrangian canonically isomorphic to the space of harmonic 1-forms on $M$. This isomorphism is seen explicitly as follows. Let $\nu \in \Gamma\left(M, N_{M / X}\right)$ be a normal vector field to $M$ in $X$. Then $\left.(\iota(\nu) \omega)\right|_{M}$ and $\left.(\iota(\nu) \operatorname{Im} \Omega)\right|_{M}$ are both seen to be well-defined forms on $M$ : one needs to lift $\nu$ to a vector field but the choice is irrelevant because $\omega$ and $\operatorname{Im} \Omega$ restrict to zero on $M$. McLean shows that if $M$ is special Lagrangian then

$$
\iota(\nu) \operatorname{Im} \Omega=-* \iota(\nu) \omega,
$$

where $*$ denotes the Hodge star operator on $M$, and furthermore, $\nu$ corresponds to an infinitesimal deformation preserving the special Lagrangian condition if and only if $d(\iota(\nu) \omega)=d(\iota(\nu) \operatorname{Im} \Omega)=0$. This gives the correspondence between harmonic 1 -forms and infinitesimal special Lagrangian deformations.

Let $f: X \rightarrow B$ be a special Lagrangian fibration with torus fibres, and assume for now that all fibres of $f$ are non-singular. Then we obtain three structures on $B$ : two affine structures and a metric, as we shall now see.

Definition 2.1. Let $B$ be an $n$-dimensional manifold. An affine structure on $B$ is given by an atlas $\left\{\left(U_{i}, \psi_{i}\right)\right\}$ of coordinate charts $\psi_{i}: U_{i} \rightarrow \mathbb{R}^{n}$, whose transition functions $\psi_{i} \circ \psi_{j}^{-1}$ lie in $\operatorname{Aff}\left(\mathbb{R}^{n}\right)$. We say the affine structure is tropical if the transition functions lie in $\mathbb{R}^{n} \rtimes G L\left(\mathbb{Z}^{n}\right)$, i.e. have integral linear part. We say the affine structure is integral if the transition functions lie in $\operatorname{Aff}\left(\mathbb{Z}^{n}\right)$.

If an affine manifold $B$ carries a Riemannian metric $g$, then we say the metric is affine Kähler or Hessian if $g$ is locally given by $g_{i j}=\partial^{2} K / \partial y_{i} \partial y_{j}$ for some convex function $K$ and $y_{1}, \ldots, y_{n}$ affine coordinates.

Then we obtain the three structures as follows:

Affine structure 1. For a normal vector field $\nu$ to a fibre $X_{b}$ of $f,\left.(\iota(\nu) \omega)\right|_{X_{b}}$ is a well-defined 1-form on $X_{b}$, and we can compute its periods as follows. Let $U \subseteq B$ be a small open set, and suppose we have submanifolds $\gamma_{1}, \ldots, \gamma_{n} \subseteq f^{-1}(U)$ which are families of 1 -cycles over $U$ and such that $\gamma_{1} \cap X_{b}, \ldots, \gamma_{n} \cap X_{b}$ form a basis for $H_{1}\left(X_{b}, \mathbb{Z}\right)$ for each $b \in U$. Consider the 1 -forms $\omega_{1}, \ldots, \omega_{n}$ on $U$ defined by 
fibrewise integration:

$$
\omega_{i}(\nu)=\int_{X_{b} \cap \gamma_{i}} \iota(\nu) \omega
$$

for $\nu$ a tangent vector on $B$ at $b$, which we can lift to a normal vector field of $X_{b}$. We have $\omega_{i}=f_{*}\left(\left.\omega\right|_{\gamma_{i}}\right)$, and since $\omega$ is closed, so is $\omega_{i}$. Thus there are locally defined functions $y_{1}, \ldots, y_{n}$ on $U$ with $d y_{i}=\omega_{i}$. Furthermore, these functions are well-defined up to the choice of basis of $H_{1}\left(X_{b}, \mathbb{Z}\right)$ and constants. Finally, they give well-defined coordinates, as follows from the fact that $\nu \mapsto \iota(\nu) \omega$ yields an isomorphism of $\mathcal{T}_{B, b}$ with $H^{1}\left(X_{b}, \mathbb{R}\right)$ by McLean's theorem. Thus $y_{1}, \ldots, y_{n}$ define local coordinates of a tropical affine structure on $B$.

Affine structure 2. We can play the same trick with $\operatorname{Im} \Omega$ : choose submanifolds $\Gamma_{1}, \ldots, \Gamma_{n} \subseteq f^{-1}(U)$ which are families of $n-1$-cycles over $U$ and such that $\Gamma_{1} \cap$ $X_{b}, \ldots, \Gamma_{n} \cap X_{b}$ form a basis for $H^{n-1}\left(X_{b}, \mathbb{Z}\right)$. We define $\lambda_{i}$ by $\lambda_{i}=-f_{*}\left(\left.\operatorname{Im} \Omega\right|_{\Gamma_{i}}\right)$, or equivalently,

$$
\lambda_{i}(\nu)=-\int_{X_{b} \cap \Gamma_{i}} \iota(\nu) \operatorname{Im} \Omega .
$$

Again $\lambda_{1}, \ldots, \lambda_{n}$ are closed 1 -forms, with $\lambda_{i}=d \check{y}_{i}$ locally, and again $\check{y}_{1}, \ldots, \check{y}_{n}$ are affine coordinates for a tropical affine structure on $B$.

The McLean metric. The Hodge metric on $H^{1}\left(X_{b}, \mathbb{R}\right)$ is given by

$$
g(\alpha, \beta)=\int_{X_{b}} \alpha \wedge * \beta
$$

for $\alpha, \beta$ harmonic 1-forms, and hence induces a metric on $B$, which can be written as

$$
g\left(\nu_{1}, \nu_{2}\right)=-\int_{X_{b}} \iota\left(\nu_{1}\right) \omega \wedge \iota\left(\nu_{2}\right) \operatorname{Im} \Omega .
$$

A crucial observation of Hitchin [30] is that these structures are related by the Legendre transform:

Proposition 2.2. Let $y_{1}, \ldots, y_{n}$ be local affine coordinates on $B$ with respect to the affine structure induced by $\omega$. Then locally there is a function $K$ on $B$ such that

$$
g\left(\partial / \partial y_{i}, \partial / \partial y_{j}\right)=\partial^{2} K / \partial y_{i} \partial y_{j}
$$

Furthermore, $\check{y}_{i}=\partial K / \partial y_{i}$ form a system of affine coordinates with respect to the affine structure induced by $\operatorname{Im} \Omega$, and if

$$
\check{K}\left(\check{y}_{1}, \ldots, \check{y}_{n}\right)=\sum \check{y}_{i} y_{i}-K\left(y_{1}, \ldots, y_{n}\right)
$$

is the Legendre transform of $K$, then

$$
y_{i}=\partial \check{K} / \partial \check{y}_{i}
$$

and

$$
\partial^{2} \check{K} / \partial y_{i} \partial y_{j}=g\left(\partial / \partial \check{y}_{i}, \partial / \partial \check{y}_{j}\right)
$$

Proof. Take families $\gamma_{1}, \ldots, \gamma_{n}, \Gamma_{1}, \ldots, \Gamma_{n}$ as above over an open neighbourhood $U$ with the two bases being Poincaré dual, i.e. $\left(\gamma_{i} \cap X_{b}\right) \cdot\left(\Gamma_{j} \cap X_{b}\right)=\delta_{i j}$ for $b \in U$. Let $\gamma_{1}^{*}, \ldots, \gamma_{n}^{*}$ and $\Gamma_{1}^{*}, \ldots, \Gamma_{n}^{*}$ be the dual bases for $\Gamma\left(U, R^{1} f_{*} \mathbb{Z}\right)$ 
and $\Gamma\left(U, R^{n-1} f_{*} \mathbb{Z}\right)$ respectively. From the choice of $\gamma_{i}$ 's, we get local coordinates $y_{1}, \ldots, y_{n}$ with $d y_{i}=\omega_{i}$, so in particular

$$
\delta_{i j}=\omega_{i}\left(\partial / \partial y_{j}\right)=\int_{\gamma_{i} \cap X_{b}} \iota\left(\partial / \partial y_{j}\right) \omega
$$

so $\iota\left(\partial / \partial y_{j}\right) \omega$ defines the cohomology class $\gamma_{j}^{*}$ in $H^{1}\left(X_{b}, \mathbb{R}\right)$. Similarly, let

$$
g_{i j}=-\int_{\Gamma_{i} \cap X_{b}} \iota\left(\partial / \partial y_{j}\right) \operatorname{Im} \Omega
$$

then $-\iota\left(\partial / \partial y_{j}\right) \operatorname{Im} \Omega$ defines the cohomology class $\sum_{i} g_{i j} \Gamma_{i}^{*}$ in $H^{n-1}\left(X_{b}, \mathbb{R}\right)$, and $\lambda_{i}=\sum_{j} g_{i j} d y_{j}$. Thus

$$
\begin{aligned}
g\left(\partial / \partial y_{j}, \partial / \partial y_{k}\right) & =-\int_{X_{b}} \iota\left(\partial / \partial y_{j}\right) \omega \wedge \iota\left(\partial / \partial y_{k}\right) \operatorname{Im} \Omega \\
& =g_{j k} .
\end{aligned}
$$

On the other hand, let $\check{y}_{1}, \ldots, \check{y}_{n}$ be coordinates with $d \check{y}_{i}=\lambda_{i}$. Then

$$
\partial \check{y}_{i} / \partial y_{j}=g_{i j}=g_{j i}=\partial \check{y}_{j} / \partial y_{i}
$$

so $\sum \check{y}_{i} d y_{i}$ is a closed 1 -form. Thus there exists locally a function $K$ such that $\partial K / \partial y_{i}=\check{y}_{i}$ and $\partial^{2} K / \partial y_{i} \partial y_{j}=g\left(\partial / \partial y_{i}, \partial / \partial y_{j}\right)$. A simple calculation then confirms that $\partial \check{K} / \partial \check{y}_{i}=y_{i}$. On the other hand,

$$
\begin{aligned}
g\left(\partial / \partial \check{y}_{i}, \partial / \partial \check{y}_{j}\right) & =g\left(\sum_{k} \frac{\partial y_{k}}{\partial \check{y}_{i}} \frac{\partial}{\partial y_{k}}, \sum_{l} \frac{\partial y_{l}}{\partial \check{y}_{j}} \frac{\partial}{\partial y_{l}}\right) \\
& =\sum_{k, l} \frac{\partial y_{k}}{\partial \check{y}_{i}} \frac{\partial y_{l}}{\partial \check{y}_{j}} g\left(\partial / \partial y_{k}, \partial / \partial y_{l}\right) \\
& =\sum_{k, l} \frac{\partial y_{k}}{\partial \check{y}_{i}} \frac{\partial y_{l}}{\partial \check{y}_{j}} \frac{\partial \check{y}_{k}}{\partial y_{l}} \\
& =\frac{\partial y_{j}}{\partial \check{y}_{i}}=\frac{\partial^{2} \check{K}}{\partial \check{y}_{i} \partial \check{y}_{j}} .
\end{aligned}
$$

Thus we introduce the notion of Legendre transform of an affine manifold with a multi-valued convex function.

Definition 2.3. Let $B$ be an affine manifold. A multi-valued function $K$ on $B$ is a collection of functions on an open cover $\left\{\left(U_{i}, K_{i}\right)\right\}$ such that on $U_{i} \cap U_{j}$, $K_{i}-K_{j}$ is affine linear. We say $K$ is convex if the Hessian $\left(\partial^{2} K_{i} / \partial y_{j} \partial y_{k}\right)$ is positive definite for all $i$, in any, or equivalently all, affine coordinate systems $y_{1}, \ldots, y_{n}$.

Given a pair $(B, K)$ of affine manifold and convex multi-valued function, the Legendre transform of $(B, K)$ is a pair $(\check{B}, \check{K})$ where $\check{B}$ is an affine structure on the underlying manifold of $B$ with coordinates given locally by $\check{y}_{i}=\partial K / \partial y_{i}$, and $\check{K}$ is defined by

$$
\check{K}_{i}\left(\check{y}_{1}, \ldots, \check{y}_{n}\right)=\sum \check{y}_{j} y_{j}-K_{i}\left(y_{1}, \ldots, y_{n}\right) .
$$

EXERCISE 2.4. Check that $\check{K}$ is also convex, and that the Legendre transform of $(\check{B}, \check{K})$ is $(B, K)$. 


\section{Semi-flat mirror symmetry}

Now let's forget about special Lagrangian fibrations for the moment. Instead, we see how the structures found on $B$ give a toy version of mirror symmetry.

Definition 3.1. Let $B$ be a tropical affine manifold.

(1) Define $\Lambda \subseteq \mathcal{T}_{B}$ to be the local system of lattices generated locally by $\partial / \partial y_{1}, \ldots, \partial / \partial y_{n}$, where $y_{1}, \ldots, y_{n}$ are local affine coordinates. This is well-defined because transition maps are in $\mathbb{R}^{n} \rtimes G L_{n}(\mathbb{Z})$. Set

$$
X(B):=\mathcal{T}_{B} / \Lambda
$$

this is a torus bundle over $B$. In addition, $X(B)$ carries a complex structure defined locally as follows. Let $U \subseteq B$ be an open set with affine coordinates $y_{1}, \ldots, y_{n}$, so $\mathcal{T}_{U}$ has coordinate functions $y_{1}, \ldots, y_{n}$, $x_{1}=d y_{1}, \ldots, x_{n}=d y_{n}$. Then

$$
q_{j}=e^{2 \pi i\left(x_{j}+i y_{j}\right)}
$$

gives a system of holomorphic coordinates on $T_{U} /\left.\Lambda\right|_{U}$, and the induced complex structure is independent of the choice of affine coordinates.

Later we will need a variant of this: for $\epsilon>0$, set

$$
X_{\epsilon}(B):=\mathcal{T}_{B} / \epsilon \Lambda
$$

this has a complex structure with coordinates given by

$$
q_{j}=e^{2 \pi i\left(x_{j}+i y_{j}\right) / \epsilon} .
$$

(As we shall see later, the limit $\epsilon \rightarrow 0$ corresponds to a large complex structure limit.)

(2) Define $\check{\Lambda} \subseteq \mathcal{T}_{B}^{*}$ to be the local system of lattices generated locally by $d y_{1}, \ldots, d y_{n}$, with $y_{1}, \ldots, y_{n}$ local affine coordinates. Set

$$
\check{X}(B):=\mathcal{T}_{B}^{*} / \check{\Lambda}
$$

Of course $\mathcal{T}_{B}^{*}$ carries a canonical symplectic structure, and this symplectic structure descends to $\check{X}(B)$.

We write $f: X(B) \rightarrow B$ and $\check{f}: \check{X}(B) \rightarrow B$ for these torus fibrations; these are clearly dual.

Now suppose in addition we have a Hessian metric $g$ on $B$, with local potential function $K$. Then in fact both $X(B)$ and $\check{X}(B)$ become Kähler manifolds:

Proposition 3.2. $K \circ f$ is a (local) Kähler potential on $X(B)$, defining a Kähler form $\omega=2 i \partial \bar{\partial}(K \circ f)$. This metric is Ricci-flat if and only if $K$ satisfies the real Monge-Ampère equation

$$
\operatorname{det} \frac{\partial^{2} K}{\partial y_{i} \partial y_{j}}=\text { constant }
$$

Proof. Working locally with affine coordinates $\left(y_{i}\right)$ and complex coordinates $z_{j}=\frac{1}{2 \pi i} \log q_{j}=x_{j}+i y_{j}$, we compute $\omega=2 i \partial \bar{\partial}(K \circ f)=\frac{i}{2} \sum \frac{\partial^{2} K}{\partial y_{j} \partial y_{k}} d z_{j} \wedge d \bar{z}_{k}$ which is clearly positive. Furthermore, if $\Omega=d z_{1} \wedge \cdots \wedge d z_{n}$, then $\omega^{n}$ is proportional to $\Omega \wedge \bar{\Omega}$ if and only if $\operatorname{det}\left(\partial^{2} K / \partial y_{j} \partial y_{k}\right)$ is constant. 
We write this Kähler manifold as $X(B, K)$.

Dually we have

Proposition 3.3. In local canonical coordinates $y_{i}, \check{x}_{i}$ on $\mathcal{T}_{B}^{*}$, the functions $z_{j}=\check{x}_{j}+i \partial K / \partial y_{j}$ on $\mathcal{T}_{B}^{*}$ induce a well-defined complex structure on $\check{X}(B)$, with respect to which the canonical symplectic form $\omega$ is a Kähler form of a metric. Furthermore this metric is Ricci-flat if and only if $K$ satisfies the real MongeAmpère equation

$$
\operatorname{det} \frac{\partial^{2} K}{\partial y_{j} \partial y_{k}}=\text { constant }
$$

Proof. It is easy to see that an affine linear change in the coordinates $y_{j}$ (and hence an appropriate change in the coordinates $\check{x}_{j}$ ) results in a linear change of the coordinates $z_{j}$, so they induce a well-defined complex structure invariant under $\check{x}_{j} \mapsto \check{x}_{j}+1$, and hence a complex structure on $\check{X}(B)$. Then one computes that

$$
\omega=\sum d \check{x}_{j} \wedge d y_{j}=\frac{i}{2} \sum g^{j k} d z_{j} \wedge d \bar{z}_{k}
$$

where $g_{i j}=\partial^{2} K / \partial y_{j} \partial y_{k}$. Then the metric is Ricci-flat if and only if $\operatorname{det}\left(g^{j k}\right)=$ constant, if and only if $\operatorname{det}\left(g_{j k}\right)=$ constant.

As before, we call this Kähler manifold $\check{X}(B, K)$.

This motivates the definition

Definition 3.4. An affine manifold with metric of Hessian form is a MongeAmpère manifold if the local potential function $K$ satisfies the Monge-Ampère equation $\operatorname{det}\left(\partial^{2} K / \partial y_{i} \partial y_{j}\right)=$ constant .

Monge-Ampère manifolds were first studied by Cheng and Yau in $\mathbf{9}$.

EXERCISE 3.5. Show that the identification of $\mathcal{T}_{B}$ and $\mathcal{T}_{B}^{*}$ given by a Hessian metric induces a canonical isomorphism $X(B, K) \cong \check{X}(\check{B}, \check{K})$ of Kähler manifolds, where $(\check{B}, \check{K})$ is the Legendre transform of $(B, K)$.

Finally, we note that a $B$-field can be introduced into this picture. To keep life relatively simple (so as to avoid having to pass to generalized complex structures 31, 27, 5]), we view the $B$-field as an element $\mathbf{B} \in H^{1}\left(B, \Lambda_{\mathbb{R}} / \Lambda\right)$, where $\Lambda_{\mathbb{R}}=$ $\Lambda \otimes_{\mathbb{Z}} \mathbb{R}$. Noting that a section of $\Lambda_{\mathbb{R}} / \Lambda$ over an open set $U$ can be viewed as a section of $\mathcal{T}_{U} /\left.\Lambda\right|_{U}$, such a section acts on $\mathcal{T}_{U} /\left.\Lambda\right|_{U}$ via translation, and this action is in fact holomorphic with respect to the standard semi-flat complex structure. Thus a Cech 1-cocycle $\left(U_{i j}, \beta_{i j}\right)$ representing $\mathbf{B}$ allows us to reglue $X(B)$ via translations over the intersections $U_{i j}$. This gives a new complex manifold $X(B, \mathbf{B})$. If in addition there is a multi-valued potential function $K$ defining a metric, these translations preserve the metric and yield a Kähler manifold $X(B, \mathbf{B}, K)$.

Thus the full toy version of mirror symmetry is as follows. The data consists of an affine manifold $B$ with potential $K$ and $B$-fields $\mathbf{B} \in H^{1}\left(B, \Lambda_{\mathbb{R}} / \Lambda\right), \check{\mathbf{B}} \in$ $H^{1}\left(B, \check{\Lambda}_{\mathbb{R}} / \check{\Lambda}\right)$. Now it is not difficult to see, and you will have seen this already if you've done Exercise 3.5, that the local system $\check{\Lambda}$ defined using the affine structure on $B$ is the same as the local system $\Lambda$ defined using the affine stucture on $\breve{B}$. So we say the pair

$$
(X(B, \mathbf{B}, K), \check{\mathbf{B}})
$$


is mirror to

$$
(X(\check{B}, \check{\mathbf{B}}, \check{K}), \mathbf{B}) \text {. }
$$

This provides a reasonably fulfilling picture of mirror symmetry in a simple context. Many more aspects of mirror symmetry can be worked out in this semiflat context, see 40]. However, ultimately this only sheds limited insight into the general case. The only compact Calabi-Yau manifolds with semi-flat Ricci-flat metric which arise in this way are complex tori (shown by Cheng and Yau in [9]). To deal with more interesting cases, we need to allow singular fibres, and hence, singularities in the affine structure of $B$.

\section{Affine manifolds with singularities}

To deal with singular fibres, we define

DEFINITION 4.1. A (tropical, integral) affine manifold with singularities is a $\left(C^{0}\right)$ manifold $B$ with an open subset $B_{0} \subseteq B$ which carries a (tropical, integral) affine structure, and such that $\Gamma:=B \backslash B_{0}$ is a locally finite union of locally closed submanifolds of codimension $\geq 2$.

By way of example, let's explain how the Batyrev construction gives rise to a wide class of such manifolds. This construction is taken from [19], where a more combinatorially complicated version is given for complete intersections; see [28] and 29 for an alternative construction.

Let $\Delta$ be a reflexive polytope in $M_{\mathbb{R}}=M \otimes_{\mathbb{Z}} \mathbb{R}$, where $M=\mathbb{Z}^{n}$; let $N$ be the dual lattice, $\nabla \subseteq N_{\mathbb{R}}$ the dual polytope given by

$$
\nabla:=\left\{n \in N_{\mathbb{R}} \mid\langle m, n\rangle \geq-1 \text { for all } m \in \Delta\right\} .
$$

We assume $0 \in \Delta$ is the unique interior lattice point of $\Delta$. Let $\check{\Sigma}$ be the normal fan to $\nabla$, consisting of cones over the faces of $\Delta$. Suppose we are given a star subdivision of $\Delta$, with all vertices being integral points, inducing a subdivision $\check{\Sigma}^{\prime}$ of the fan $\check{\Sigma}$. In addition suppose that

$$
\check{h}: M_{\mathbb{R}} \rightarrow \mathbb{R}
$$

is an (upper) strictly convex piecewise linear function on the fan $\check{\Sigma}^{\prime}$. Also, let

$$
\check{\varphi}: M_{\mathbb{R}} \rightarrow \mathbb{R}
$$

be the piecewise linear function representing the anti-canonical class of the toric variety $\mathbb{P}_{\nabla}$; i.e. $\check{\varphi}$ takes the value 1 on the primitive generator of each one-dimensional cone of $\check{\Sigma}$. Finally, assume that $\check{h}$ is chosen so that $\check{h}^{\prime}=\check{h}-\check{\varphi}$ is a (not necessarily strictly) convex function.

Define, for any convex piecewise linear function $\check{g}$ on the fan $\check{\Sigma}^{\prime}$, the Newton polytope of $\check{g}$,

$$
\nabla^{\check{g}}:=\left\{n \in N_{\mathbb{R}} \mid\langle m, n\rangle \geq-\check{g}(m) \text { for all } m \in M_{\mathbb{R}}\right\} \text {. }
$$

In particular,

$$
\nabla^{\check{h}}=\nabla^{\check{h}^{\prime}}+\nabla^{\check{\varphi}}=\nabla^{\check{h}^{\prime}}+\nabla,
$$

where + denotes Minkowski sum. Our goal will be to put an affine structure with singularities on $B:=\partial \nabla^{\breve{h}}$. Our first method of doing this requires no choices. Let $\mathscr{P}$ be the set of proper faces of $\nabla^{\breve{h}}$. Furthermore, let $\operatorname{Bar}(\mathscr{P})$ denote the first barycentric subdivision of $\mathscr{P}$ and let $\Gamma \subseteq B$ be the union of all simplices of $\operatorname{Bar}(\mathscr{P})$ not containing a vertex of $\mathscr{P}$ (a zero-dimensional cell) or intersecting the interior of 
a maximal cell of $\mathscr{P}$. If we then set $B_{0}:=B \backslash \Gamma$, we can define an affine structure on $B_{0}$ as follows. $B_{0}$ has an open cover

$$
\left\{W_{\sigma} \mid \sigma \in \mathscr{P} \text { maximal }\right\} \cup\left\{W_{v} \mid v \in \mathscr{P} \text { a vertex }\right\}
$$

where $W_{\sigma}=\operatorname{Int}(\sigma)$, the interior of $\sigma$, and

$$
W_{v}=\bigcup_{\substack{\tau \in \operatorname{Bar}(\mathscr{P}) \\ v \in \tau}} \operatorname{Int}(\tau)
$$

is the (open) star of $v$ in $\operatorname{Bar}(\mathscr{P})$. We define an affine chart

$$
\psi_{\sigma}: W_{\sigma} \rightarrow \mathbb{A}^{n-1} \subseteq N_{\mathbb{R}}
$$

given by the inclusion of $W_{\sigma}$ in $\mathbb{A}^{n-1}$, the affine hyperplane containing $\sigma$. Also, take

$$
\psi_{v}: W_{v} \rightarrow N_{\mathbb{R}} / \mathbb{R} v^{\prime}
$$

to be the projection, where $v$, being a vertex of $\nabla^{\breve{h}}$, can be written uniquely as $v^{\prime}+v^{\prime \prime}$ with $v^{\prime}$ a vertex of $\nabla$ and $v^{\prime \prime}$ a vertex of $\nabla^{\check{h}^{\prime}}$. One checks easily that for $v \in \sigma, \psi_{\sigma} \circ \psi_{v}^{-1}$ is affine linear with integral linear part (integrality follows from reflexivity of $\Delta$ !) so $B$ is a tropical affine manifold with singularities. Furthermore, if $\breve{h}$ was chosen to have integral slopes, then $B$ is integral.

We often would like to refine this construction, to get a finer polyhedral decomposition $\mathscr{P}$ of $B$ and with it a somewhat more interesting discriminant locus $\Gamma$. One reason for doing so is that this construction is clearly not mirror symmetric, as it depends only on a star subdivision of $\Delta$ and not of $\nabla$. Furthermore, a maximal star subdivision of $\nabla$ corresponds to what Batyrev terms a MPCP (maximal projective crepant partial) resolution of $\mathbb{P}_{\Delta}$, and normally, we will wish to study hypersurfaces in a MPCP resolution of $\mathbb{P}_{\Delta}$ rather than in $\mathbb{P}_{\Delta}$ itself. To introduce this extra degree of flexibility, we need to make some choices, which is done as follows.

First, choose a star subdivision of $\nabla$, with all vertices being integral points, inducing a refinement $\Sigma^{\prime}$ of the fan $\Sigma$ which is the normal fan to $\Delta$. This induces a polyhedral subdivision of $\partial \nabla$, and we write the collection of cells of this subdivision as $\mathscr{P}_{\partial \nabla}$. Note that because $0 \in \nabla$, we have

$$
\nabla^{\check{h}^{\prime}} \subseteq \nabla^{\check{h}^{\prime}}+\nabla=\nabla^{\check{h}} .
$$

Definition 4.2. A subdivision $\mathscr{P}$ of $\partial \nabla^{\check{h}}$ is good with respect to $\mathscr{P}_{\partial \nabla}$ if it is induced by a subdivision $\mathscr{P}_{\nabla_{\check{h}}}$ of $\nabla^{\breve{h}}$ satisfying the following three properties:

(1) $\nabla^{\check{h}^{\prime}}$ is a union of cells in $\mathscr{P}_{\nabla \check{h}}$.

(2) All vertices of $\mathscr{P}_{\nabla \check{h}}$ are contained either in $\partial \nabla^{\check{h}}$ or in $\nabla^{\check{h}}$.

(3) Every cell $\sigma \in \mathscr{P}_{\nabla^{\check{h}}}$ with $\sigma \cap \partial \nabla^{\check{h}} \neq \emptyset$ and $\tau:=\sigma \cap \partial \nabla^{\check{h}^{\prime}} \neq \emptyset$ can be written as

$$
\sigma=\left(C\left(\sigma^{\prime}\right)+\tau\right) \cap \nabla^{\check{h}},
$$

with $\sigma^{\prime} \in \mathscr{P}_{\partial \nabla}$ and $C\left(\sigma^{\prime}\right)$ the corresponding cone in $\Sigma^{\prime}$.

If $\check{h}$ has integral slopes and all vertices of $\mathscr{P}_{\nabla \check{h}}$ are integral, then we say $\mathscr{P}$ is integral. 
The following picture shows what such a good subdivision may look like, in the case that $\nabla$ is the Newton polytope of $\mathcal{O}_{\mathbb{P}^{2}}(3)$ :

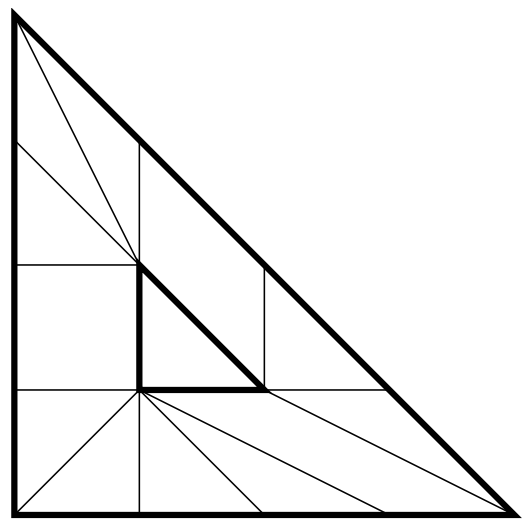

Given a good decomposition $\mathscr{P}$ of

$$
B:=\partial \nabla^{\check{h}},
$$

we once again obtain an affine structure with singularities on $B$, much as before, defining the discriminant locus $\Gamma \subseteq B$ in terms of the first barycentric subdivision $\operatorname{Bar}(\mathscr{P})$ of this new polyhedral decomposition $\mathscr{P}$. Then as before $B_{0}:=B \backslash \Gamma$ has an open cover

$$
\left\{W_{\sigma} \mid \sigma \in \mathscr{P} \text { maximal }\right\} \cup\left\{W_{v} \mid v \in \mathscr{P} \text { a vertex }\right\}
$$

where $W_{\sigma}=\operatorname{Int}(\sigma)$, the interior of $\sigma$, and

$$
W_{v}=\bigcup_{\substack{\tau \in \operatorname{Bar}(\mathscr{P}) \\ v \in \tau}} \operatorname{Int}(\tau)
$$

is the (open) star of $v$ in $\operatorname{Bar}(\mathscr{P})$. We define an affine chart

$$
\psi_{\sigma}: W_{\sigma} \rightarrow \mathbb{A}^{n-1} \subseteq N_{\mathbb{R}}
$$

given by the inclusion of $W_{\sigma}$ in $\mathbb{A}^{n-1}$, the affine hyperplane containing $\sigma$. Also, take $\psi_{v}: W_{v} \rightarrow N_{\mathbb{R}} / \mathbb{R} v^{\prime}$ to be the projection, where $v$ can be written uniquely as $v^{\prime}+v^{\prime \prime}$ with $v^{\prime}$ an integral point of $\nabla$ and $v^{\prime \prime} \in \nabla^{\breve{h}^{\prime}}$. As before, one checks easily that for $v \in \sigma, \psi_{\sigma} \circ \psi_{v}^{-1}$ is affine linear with integral linear part so $B$ is a tropical affine manifold with singularities. Furthermore, if $\breve{h}$ has integral slopes, and $\mathscr{P}$ is integral, then the affine structure on $B$ is in fact integral.

EXAMPLE 4.3. Let $\Delta \subseteq \mathbb{R}^{4}$ be the convex hull of the points

$$
(-1,-1,-1,-1),(1,0,0,0),(0,1,0,0),(0,0,1,0),(0,0,0,1),
$$

so $\nabla$ is the convex hull of the points

$$
(-1,-1,-1,-1),(4,-1,-1,-1),(-1,4,-1,-1),(-1,-1,4,-1),(-1,-1,-1,4) \text {. }
$$

Take $\check{h}=\check{\varphi}$ and choose a star triangulation of $\nabla$. In this case $B=\partial \nabla$. It is easy to see the affine structure on $B_{0}$ in fact extends across the interior of all threedimensional faces of $\nabla$. This gives a smaller discriminant locus $\Gamma$ which, given a nice regular triangulation of $\nabla$, looks like the following picture in a neighbourhood of a 2 -face of $\nabla$ : the light lines giving the triangulation and the dark lines the discriminant locus $\Gamma$. 


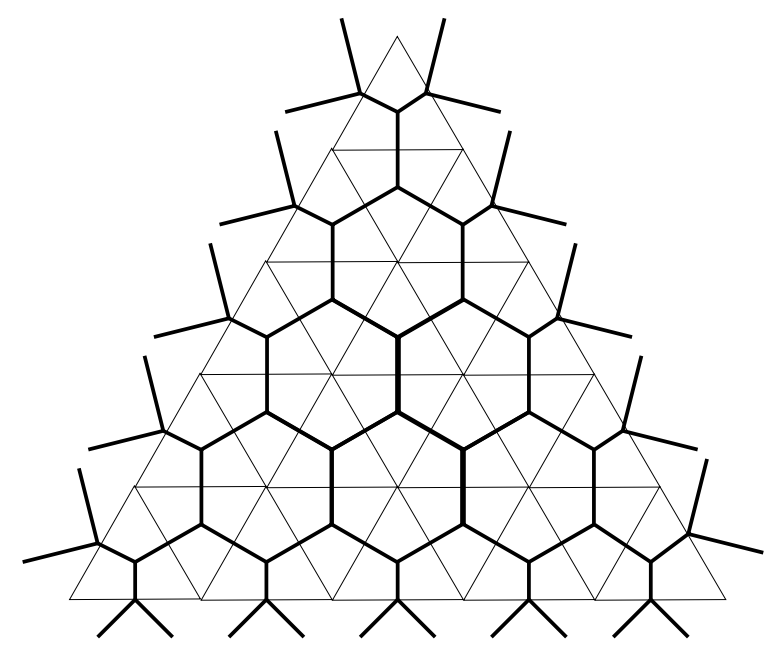

In this picture, $\Gamma$ is a trivalent graph, with two types of trivalent vertices. The ones along the edge are non-planar: the two additional legs of $\Gamma$ drawn in this picture are contained in other two-faces of $\nabla$. However $\Gamma$ is planar in the interior of this two-face.

In general, if the subdivisions $\Sigma^{\prime}$ and $\check{\Sigma}^{\prime}$ of $\Sigma$ and $\check{\Sigma}$ respectively represent maximal projective crepant partial resolutions of $\mathbb{P}_{\Delta}$ and $\mathbb{P}_{\nabla}$, and $\operatorname{dim} B=3$, then only these sorts of trivalent vertices occur. More specifically, one knows what the monodromy of the local systems $\Lambda$ and $\check{\Lambda}$ on $B_{0}$ look like at these vertices. If $v \in \Gamma$ is a vertex contained in the interior of a two-face, then it is clear that the tangent space to that two-face is invariant under parallel transport, in a neighbourhood of $v$, of $\Lambda$. A more careful analysis yields that the monodromy matrices for $\Lambda$ take the form, in a suitable basis,

$$
T_{1}=\left(\begin{array}{lll}
1 & 0 & 0 \\
1 & 1 & 0 \\
0 & 0 & 1
\end{array}\right), T_{2}=\left(\begin{array}{lll}
1 & 0 & 0 \\
0 & 1 & 0 \\
1 & 0 & 1
\end{array}\right), T_{3}=\left(\begin{array}{ccc}
1 & 0 & 0 \\
-1 & 1 & 0 \\
-1 & 0 & 1
\end{array}\right)
$$

Here $T_{1}, T_{2}, T_{3}$ are given by parallel transport about loops around the three edges of $\Gamma$ coming out of $v$. Of course, the monodromy of $\check{\Lambda}$ is the transpose inverse of these matrices. Similarly, if $v$ is a vertex of $\Gamma$ contained in an edge of $\nabla^{\breve{h}}$, then the monodromy will take the form

$$
T_{1}=\left(\begin{array}{ccc}
1 & -1 & 0 \\
0 & 1 & 0 \\
0 & 0 & 1
\end{array}\right), T_{2}=\left(\begin{array}{ccc}
1 & 0 & -1 \\
0 & 1 & 0 \\
0 & 0 & 1
\end{array}\right), T_{3}=\left(\begin{array}{lll}
1 & 1 & 1 \\
0 & 1 & 0 \\
0 & 0 & 1
\end{array}\right) .
$$

So we see that the monodromy of the two types of vertices are interchanged by looking at $\Lambda$ and $\check{\Lambda}$.

One main result of [17] is

THEOREM 4.4. If B is a three-dimensional tropical affine manifold with singularities such that $\Gamma$ is trivalent and the monodromy of $\Lambda$ at each vertex is one of the above two types, then $f_{0}: X\left(B_{0}\right) \rightarrow B_{0}$ can be compactified to a topological fibration $f: X(B) \rightarrow B$. Dually, $\check{f}_{0}: \check{X}\left(B_{0}\right) \rightarrow B_{0}$ can be compactified to a topological fibration $\check{f}: \check{X}(B) \rightarrow B$. 
We won't give any details here of how this is carried out, but it is not particularly difficult, as long as one restricts to the category of topological (not $C^{\infty}$ ) manifolds. However, it is interesting to look at the singular fibres we need to add in this compactification.

If $b \in \Gamma$ is a point which is not a vertex of $\Gamma$, then $f^{-1}(b)$ is homeomorphic to $I_{1} \times S^{1}$, where $I_{1}$ denotes a Kodaira type $I_{1}$ elliptic curve, i.e. a pinched torus.

If $b$ is a vertex of $\Gamma$, with monodromy of the first type, then $f^{-1}(b)=S^{1} \times$ $S^{1} \times S^{1} / \sim$, with $(a, b, c) \sim\left(a^{\prime}, b^{\prime}, c^{\prime}\right)$ if $(a, b, c)=\left(a^{\prime}, b^{\prime}, c^{\prime}\right)$ or $a=a^{\prime}=1$, where $S^{1}$ is identified with the unit circle in $\mathbb{C}$. This is the three-dimensional analogue of a pinched torus, and $\chi\left(f^{-1}(b)\right)=+1$. We call this a positive fibre.

If $b$ is a vertex of $\Gamma$, with monodromy of the second type, then $f^{-1}(b)$ can be described as $S^{1} \times S^{1} \times S^{1} / \sim$, with $(a, b, c) \sim\left(a^{\prime}, b^{\prime}, c^{\prime}\right)$ if $(a, b, c)=\left(a^{\prime}, b^{\prime}, c^{\prime}\right)$ or $a=a^{\prime}=1, b=b^{\prime}$, or $a=a^{\prime}, b=b^{\prime}=1$. The singular locus of this fibre is a figure eight, and $\chi\left(f^{-1}(b)\right)=-1$. We call this a negative fibre.

So we see a very concrete local consequence of SYZ duality: namely in the compactifications $X(B)$ and $\check{X}(B)$, the positive and negative fibres are interchanged. Of course, this results in the observation that Euler characteristic changes sign under mirror symmetry for Calabi-Yau threefolds.

EXAMPLE 4.5. Continuing with Example 4.3, it was proved in 17 that $\check{X}(B)$ is homeomorphic to the quintic and $X(B)$ is homeomorphic to the mirror quintic.

REMARK 4.6. Haase and Zharkov in $\mathbf{2 8}$ gave a different description of what is the same affine structure. Their construction has the advantage that it is manifestly dual. In other words, in our construction, we can interchange the role of $\Delta$ and $\nabla$ to get two different affine manifolds, with $B_{\Delta}$ the affine manifold with singularities structure on $\partial \Delta^{h}$ and $B_{\nabla}$ the affine manifold with singularities structure on $\partial \nabla^{\check{h}}$. It is not obvious that these are "dual" affine manifolds, at least in the sense that $X\left(B_{\nabla}\right)$ is homeomorphic to $\check{X}\left(B_{\Delta}\right)$ and $\check{X}\left(B_{\nabla}\right)$ is homeomorphic to $X\left(B_{\Delta}\right)$. In the construction given above, this follows from the discrete Legendre transform we will discuss in $\S 7$. On the other hand, the construction I give here will arise naturally from the degeneration construction discussed later in this paper.

Ruan in $\mathbf{5 2}$ gave a description of Lagrangian torus fibrations for hypersurfaces in toric varieties using a symplectic flow argument, and his construction should coincide with a symplectic compactification of the symplectic manifolds $\check{X}\left(B_{0}\right)$. In the three-dimensional case, such a symplectic compactification has now been constructed by Ricardo Castaño-Bernard and Diego Matessi [7. If this compactification is applied to the affine manifolds with singularities described here, the resulting symplectic manifolds should be symplectomorphic to the corresponding toric hypersurface, but this has not yet been shown.

I should also point out that the explicit compactifications mentioned in three dimensions can be carried out in all dimensions, and will be done so in [21. We will show there in a much more general context that these compactifications are then homeomorphic to the expected Calabi-Yau manifolds.

\section{The problems with the SYZ conjecture, and how to get around them}

The previous section demonstrates that the SYZ conjecture gives a beautiful description of mirror symmetry at a purely topological level. This, by itself, can often be useful, but unfortunately is not strong enough to get at really interesting 
aspects of mirror symmetry, such as instanton corrections. For a while, though, many of us were hoping that the strong version of duality we have just seen would hold at the special Lagrangian level. This would mean that a mirror pair $X, \check{X}$ would possess special Lagrangian torus fibrations $f: X \rightarrow B$ and $\check{f}: \check{X} \rightarrow B$ with codimension two discriminant locus, and the discriminant loci of $f$ and $\check{f}$ would coincide. These fibrations would then be dual away from the discriminant locus.

There are examples of special Lagrangian fibrations on non-compact toric varieties $X$ with this behaviour. In particular, if $\operatorname{dim} X=n$ with a $T^{n-1}$ action on $X$ preserving the holomorphic $n$-form, and if $X$ in addition carries a Ricci-flat metric which is invariant under this action, then $X$ will have a very nice special Lagrangian fibration with codimension two discriminant locus. (See [18 and [14]). However, Dominic Joyce (33 and other papers cited therein) began studying some threedimensional $S^{1}$-invariant examples, and discovered quite different behaviour. There is an argument that if a special Lagrangian fibration is $C^{\infty}$, then the discriminant locus will be (Hausdorff) codimension two. However, Joyce discovered examples which were not differentiable, but only piecewise differentiable, and furthermore, had a codimension one discriminant locus:

Example 5.1. Define $F: \mathbb{C}^{3} \rightarrow \mathbb{R} \times \mathbb{C}$ by $F\left(z_{1}, z_{2}, z_{3}\right)=(a, c)$ with $2 a=$ $\left|z_{1}\right|^{2}-\left|z_{2}\right|^{2}$ and

$$
c= \begin{cases}z_{3} & a=z_{1}=z_{2}=0 \\ z_{3}-\bar{z}_{1} \bar{z}_{2} /\left|z_{1}\right| & a \geq 0, z_{1} \neq 0 \\ z_{3}-\bar{z}_{1} \bar{z}_{2} /\left|z_{2}\right| & a<0\end{cases}
$$

It is easy to see that if $a \neq 0$, then $F^{-1}(a, c)$ is homeomorphic to $\mathbb{R}^{2} \times S^{1}$, while if $a=0$, then $F^{-1}(a, c)$ is a cone over $T^{2}$ : essentially, one copy of $S^{1}$ in $\mathbb{R}^{2} \times S^{1}$ collapses to a point. In addition, all fibres of this map are special Lagrangian, and it is obviously only piecewise smooth. The discriminant locus is the entire plane given by $a=0$.

This example forces a reevaluation of the strong form of the SYZ conjecture. In further work Joyce found evidence for a more likely picture for general special Lagrangian fibrations in three dimensions. The discriminant locus, instead of being a codimension two graph, will be a codimension one blob. Typically the union of the singular points of singular fibres will be a Riemann surface, and it will map to an amoeba shaped set in $B$, i.e. the discriminant locus looks like the picture on the right rather than the left, and will be a fattening of the old picture of a codimension two discriminant. 

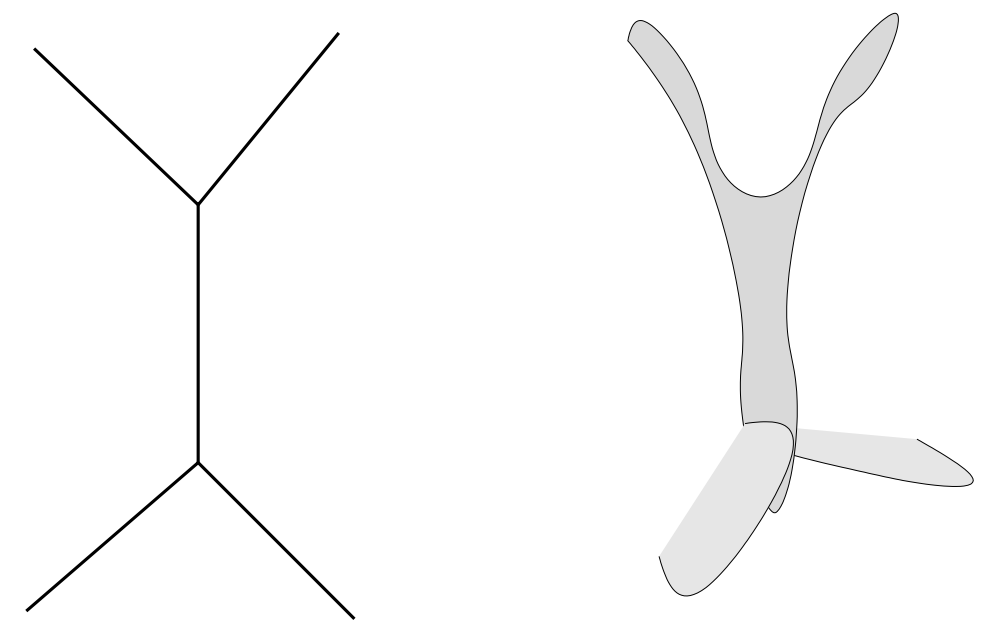

Joyce made some additional arguments to suggest that this fattened discriminant locus must look fundamentally different in a neighbourhood of the two basic types of vertices we saw in the previous section, with the two types of vertices expected to appear pretty much as depicted in the above picture. Thus the strong form of duality mentioned above, where we expect the discriminant loci of the special Lagrangian fibrations on a mirror pair to be the same, cannot hold. If this is the case, one needs to replace this strong form of duality with a weaker form.

It seems likely that the best way to rephrase the SYZ conjecture is in a limiting form. Mirror symmetry as we currently understand it has to do with degenerations of Calabi-Yau manifolds. Given a flat family $f: \mathcal{X} \rightarrow D$ over a disk $D$, with the fibre $\mathcal{X}_{0}$ over 0 singular and all other fibres $n$-dimensional Calabi-Yau manifolds, we say the family is maximally unipotent if the monodromy transformation $T: H^{n}\left(\mathcal{X}_{t}, \mathbb{Q}\right) \rightarrow H^{n}\left(\mathcal{X}_{t}, \mathbb{Q}\right)(t \in D$ non-zero $)$ satisfies $(T-I)^{n+1}=0$ but $(T-I)^{n} \neq 0$. It is a standard fact of mirror symmetry that mirrors should be associated to maximally unipotent degenerations of Calabi-Yau manifolds. In particular, given two different maximally unipotent degenerations in a single complex moduli space for some Calabi-Yau manifold, one might obtain different mirror manifolds. Sometimes these different mirror manifolds are birationally equivalent, as studied in [1], or are genuinely different, see [51.

We recall the definition of Gromov-Hausdorff convergence, a notion of convergence of a sequence of metric spaces.

Definition 5.2. Let $\left(X, d_{X}\right),\left(Y, d_{Y}\right)$ be two compact metric spaces. Suppose there exists maps $f: X \rightarrow Y$ and $g: Y \rightarrow X$ (not necessarily continuous) such that for all $x_{1}, x_{2} \in X$,

$$
\left|d_{X}\left(x_{1}, x_{2}\right)-d_{Y}\left(f\left(x_{1}\right), f\left(x_{2}\right)\right)\right|<\epsilon
$$

and for all $x \in X$,

$$
d_{X}(x, g \circ f(x))<\epsilon,
$$

and the two symmetric properties for $Y$ hold. Then we say the Gromov-Hausdorff distance between $X$ and $Y$ is at most $\epsilon$. The Gromov-Hausdorff distance $d_{G H}(X, Y)$ is the infimum of all such $\epsilon$.

It follows from results of Gromov (see for example [49, pg. 281, Cor. 1.11) that the space of compact Ricci-flat manifolds with diameter $\leq C$ is precompact 
with respect to Gromov-Hausdorff distance, i.e. any sequence of such manifolds has a subsequence converging with respect to the Gromov-Hausdorff distance to a metric space. This metric space could be quite bad; this is quite outside the realm of algebraic geometry! Nevertheless, this raises the following natural question. Given a maximally unipotent degeneration of Calabi-Yau manifolds $\mathcal{X} \rightarrow D$, take a sequence $t_{i} \in D$ converging to 0 , and consider a sequence $\left(\mathcal{X}_{t_{i}}, g_{t_{i}}\right)$, where $g_{t_{i}}$ is a choice of Ricci-flat metric chosen so that $\operatorname{Diam}\left(g_{t_{i}}\right)$ remains bounded. What is the Gromov-Hausdorff limit of $\left(\mathcal{X}_{t_{i}}, g_{t_{i}}\right)$, or the limit of some convergent subsequence?

EXAMPLE 5.3. Consider a degenerating family of elliptic curves, with periods 1 and $\frac{1}{2 \pi i} \log t$. If we take $t$ approaching 0 along the positive real axis, then we can just view this as a family of elliptic curves $\mathcal{X}_{\alpha}$ with period 1 and $i \alpha$ with $\alpha \rightarrow \infty$. If we take the standard Euclidean metric $g$ on $\mathcal{X}_{\alpha}$, then the diameter of $\mathcal{X}_{\alpha}$ is unbounded. To obtain a bounded diameter, we replace $g$ by $g / \alpha^{2}$; equivalently, we can keep $g$ fixed on $\mathbb{C}$ but change the periods of the elliptic curve to $1 / \alpha, i$. It then becomes clear that the Gromov-Hausdorff limit of such a sequence of elliptic curves is a circle $\mathbb{R} / \mathbb{Z}$.

This simple example motivates the first conjecture about maximally unipotent degenerations, conjectured independently by myself and Wilson on the one hand [26] and Kontsevich and Soibelman [38] on the other.

ConjeCture 5.4. Let $\mathcal{X} \rightarrow D$ be a maximally unipotent degeneration of simplyconnected Calabi-Yau manifolds with full $S U(n)$ holonomy, $t_{i} \in D$ with $t_{i} \rightarrow 0$, and let $g_{i}$ be a Ricci-flat metric on $\mathcal{X}_{t_{i}}$ normalized to have fixed diameter $C$. Then a convergent subsequence of $\left(\mathcal{X}_{t_{i}}, g_{i}\right)$ converges to a metric space $\left(X_{\infty}, d_{\infty}\right)$, where $X_{\infty}$ is homeomorphic to $S^{n}$. Furthermore, $d_{\infty}$ is induced by a Riemannian metric on $X_{\infty} \backslash \Gamma$, where $\Gamma \subseteq X_{\infty}$ is a set of codimension two.

Here the topology of the limit depends on the nature of the non-singular fibres $\mathcal{X}_{t}$; for example, if instead $\mathcal{X}_{t}$ was hyperkähler, then we would expect the limit to be a projective space. Also, even in the case of full $S U(n)$ holonomy, if $\mathcal{X}_{t}$ is not simply connected, we would expect limits such as $\mathbb{Q}$-homology spheres to arise.

Conjecture 5.4 is directly inspired by the SYZ conjecture. Suppose we had special Lagrangian fibrations $f_{i}: \mathcal{X}_{t_{i}} \rightarrow B_{i}$. Then as the maximally unipotent degeneration is approached, it is possible to see that the volume of the fibres of these fibrations go to zero. This would suggest these fibres collapse, hopefully leaving the base as the limit.

This conjecture was proved by myself and Wilson for K3 surfaces in 26. The proof relies on a detailed analysis of the behaviour of Ricci-flat metrics in the limit, and also on the existence of explicit local models for Ricci-flat metrics near singular fibres of special Lagrangian fibrations.

The motivation for this conjecture from SYZ also provides a limiting form of the conjecture. There are any number of problems with trying to prove the existence of special Lagrangian fibrations on Calabi-Yau manifolds. Even the existence of a single special Lagrangian torus near a maximally unipotent degeneration is unknown, but we expect it should be easier to find them as we approach the maximally unipotent point. Furthermore, even if we find a special Lagrangian torus, we know that it moves in an $n$-dimensional family, but we don't know its deformations fill out the entire manifold. In addition, there is no guarantee that even if it does, we obtain a foliation of the manifold: nearby special Lagrangian submanifolds may 
intersect. (For an example, see [4].) So instead, we will just look at the moduli space of special Lagrangian tori.

Suppose, given $t_{i} \rightarrow 0$, that for $t_{i}$ sufficiently close to zero, there is a special Lagrangian $T^{n}$ whose homology class is invariant under monodromy, or more specifically, generates the space $W_{0}$ of the monodromy weight filtration (this is where we expect to find fibres of a special Lagrangian fibration associated to a maximally unipotent degeneration). Let $B_{0, i}$ be the moduli space of deformations of this torus; every point of $B_{0, i}$ corresponds to a smooth special Lagrangian torus in $\mathcal{X}_{t_{i}}$. This manifold then comes equipped with the McLean metric and affine structures defined in $\S 2$. One can then compactify $B_{0, i} \subseteq B_{i}$, (probably by taking the closure of $B_{0, i}$ in the space of special Lagrangian currents; the details aren't important here). This gives a series of metric spaces $\left(B_{i}, d_{i}\right)$ with the metric $d_{i}$ induced by the McLean metric. If the McLean metric is normalized to keep the diameter of $B_{i}$ constant independent of $i$, then we can hope that $\left(B_{i}, d_{i}\right)$ converges to a compact metric space $\left(B_{\infty}, d_{\infty}\right)$. Here then is the limiting form of SYZ:

ConjeCture 5.5. If $\left(\mathcal{X}_{t_{i}}, g_{i}\right)$ converges to $\left(X_{\infty}, g_{\infty}\right)$ and $\left(B_{i}, d_{i}\right)$ is non-empty for large $i$ and converges to $\left(B_{\infty}, d_{\infty}\right)$, then $B_{\infty}$ and $X_{\infty}$ are isometric up to scaling. Furthermore, there is a subspace $B_{\infty, 0} \subseteq B_{\infty}$ with $\Gamma:=B_{\infty} \backslash B_{\infty, 0}$ of Hausdorff codimension 2 in $B_{\infty}$ such that $B_{\infty, 0}$ is a Monge-Ampère manifold, with the MongeAmpère metric inducing $d_{\infty}$ on $B_{\infty, 0}$.

Essentially what this is saying is that as we approach the maximally unipotent degeneration, we expect to have a special Lagrangian fibration on larger and larger subsets of $\mathcal{X}_{t_{i}}$. Furthermore, in the limit, the codimension one discriminant locus suggested by Joyce converges to a codimension two discriminant locus, and (the not necessarily Monge-Ampère, see [44]) Hessian metrics on $B_{0, i}$ converge to a Monge-Ampère metric.

The main point I want to get at here is that it is likely the SYZ conjecture is only "approximately" correct, and one needs to look at the limit to have a hope of proving anything. On the other hand, the above conjecture seems likely to be accessible by currently understood techniques, though with a lot of additional work, and I wouldn't be surprised to see it proved in the next few years.

How do we do mirror symmetry using this modified version of the SYZ conjecture? Essentially, we would follow these steps:

(1) We begin with a maximally unipotent degeneration of Calabi-Yau manifolds $\mathcal{X} \rightarrow D$, along with a choice of polarization. This gives us a Kähler class $\left[\omega_{t}\right] \in H^{2}\left(\mathcal{X}_{t}, \mathbb{R}\right)$ for each $t \in D \backslash 0$, represented by $\omega_{t}$ the Kähler form of a Ricci-flat metric $g_{t}$.

(2) Identify the Gromov-Hausdorff limit of a sequence $\left(\mathcal{X}_{t_{i}}, r_{i} g_{t_{i}}\right)$ with $t_{i} \rightarrow 0$, and $r_{i}$ a scale factor which keeps the diameter of $\mathcal{X}_{t_{i}}$ constant. The limit will be, if the above conjectures work, an affine manifold with singularities $B$ along with a Monge-Ampère metric.

(3) Perform a Legendre transform to obtain a new affine manifold with singularities $\check{B}$, though with the same metric.

(4) Try to construct a compactification of $X_{\epsilon}\left(\check{B}_{0}\right)$ for small $\epsilon>0$ to obtain a complex manifold $X_{\epsilon}(\check{B})$. This will be the mirror manifold.

Actually, we need to elaborate on this last step a bit more. The problem is that while we expect that it should be possible in general to construct symplectic 
compactifications of the symplectic manifold $\check{X}\left(B_{0}\right)$ (and hence get the mirror as a symplectic manifold), we don't expect to be able to compactify $X_{\epsilon}\left(\check{B}_{0}\right)$ as a complex manifold. Instead, the expectation is that a small deformation of $X_{\epsilon}\left(\check{B}_{0}\right)$ is necessary before it can be compactified. Furthermore, this small deformation is critically important in mirror symmetry: it is this small deformation which provides the B-model instanton corrections.

Because of the importance of this last issue, it has already been studied by several authors: Fukaya in $\mathbf{1 1}$ has studied the problem directly using heuristic ideas, while Kontsevich and Soibelmann 39 have modified the problem of passing from an affine manifold to a complex manifold by instead producing a non-Archimedean space. We will return to these issues later in this paper, when I discuss my own work with Siebert which has been partly motivated by the same problem. Because this last item is so important, let's give it a name:

Question 5.6 (The reconstruction problem, Version I). Given a tropical affine manifold with singularities $B$, construct a complex manifold $X_{\epsilon}(B)$ which is a compactification of a small deformation of $X_{\epsilon}\left(B_{0}\right)$.

I do not wish to dwell further on this version of the SYZ conjecture here, because it lies mostly in the realm of analysis and differential geometry and the behaviour of Ricci-flat metrics, and will give us little insight into what makes subtler aspects of traditional mirror symmetry work: for example, how exactly do instanton corrections arise? So we move on to explain how the limiting form of SYZ inspired a more algebro-geometric form of SYZ, which in turn avoids all analytic problems and holds out great promise for understanding the fundamental mysteries of mirror symmetry.

\section{Gromov-Hausdorff limits, algebraic degenerations, and mirror symmetry}

We now have two notions of limit: the familiar algebro-geometric notion of a flat degenerating family $\mathcal{X} \rightarrow D$ over a disk on the one hand, and the GromovHausdorff limit on the other. Kontsevich had an important insight (see [38]) into the connection between these two. In this section I will give a rough idea of how and why this works.

Very roughly speaking, the Gromov-Hausdorff limit $\left(\mathcal{X}_{t_{i}}, g_{t_{i}}\right)$ as $t_{i} \rightarrow 0$ should coincide, topologically, with the dual intersection complex of the singular fibre $\mathcal{X}_{0}$. More precisely, in a relatively simple situation, suppose $f: \mathcal{X} \rightarrow D$ is relatively minimal (in the sense of Mori) and normal crossings, with $\mathcal{X}_{0}$ having irreducible components $X_{1}, \ldots, X_{m}$. The dual intersection complex of $\mathcal{X}_{0}$ is the simplicial complex with vertices $v_{1}, \ldots, v_{m}$, and which contains a simplex $\left\langle v_{i_{0}}, \ldots, v_{i_{p}}\right\rangle$ if $X_{i_{0}} \cap \cdots \cap X_{i_{p}} \neq \emptyset$.

Let us explain roughly why this should be, first by looking at a standard family of degenerating elliptic curves with periods 1 and $\frac{n}{2 \pi i} \log t$ for $n$ a positive integer. Such a family over the punctured disk is extended to a family over the disk by adding an $I_{n}$ (a cycle of $n$ rational curves) fibre over the origin.

Taking a sequence $t_{i} \rightarrow 0$ with $t_{i}$ real and positive gives a sequence of elliptic

curves of the form $X_{\epsilon_{i}}(B)$ where: $B=\mathbb{R} / n \mathbb{Z}$ and $\epsilon_{i}=-\frac{2 \pi}{\ln t_{i}}$. In addition, the metric on $X_{\epsilon_{i}}(B)$, properly scaled, comes from the constant Hessian metric on $B$. So we wish to explain how $B$ is related to the geometry near the singular fibre. To 
this end, let $X_{1}, \ldots, X_{n}$ be the irreducible components of $\mathcal{X}_{0}$; these are all $\mathbb{P}^{1}$ 's. Let $P_{1}, \ldots, P_{n}$ be the singular points of $\mathcal{X}_{0}$.

We'll consider two sorts of open sets in $\mathcal{X}$. For the first type, choose a coordinate $z$ on $X_{i}$, with $P_{i}$ given by $z=0$ and $P_{i+1}$ given by $z=\infty$. Let $U_{i} \subseteq D_{i}$ be the open set $\{z|\delta \leq| z \mid \leq 1 / \delta\}$ for some small fixed $\delta$. Then one can find a neighbourhood $\tilde{U}_{i}$ of $U_{i}$ in $\mathcal{X}$ such that $\tilde{U}_{i}$ is biholomorphic to $U_{i} \times D_{\rho}$ for $\rho>0$ sufficiently small, $D_{\rho}$ a disk of radius $\rho$ in $\mathbb{C}$, and $\left.f\right|_{\tilde{U}_{i}}$ is the projection onto $D_{\rho}$.

On the other hand, each $P_{i}$ has a neighbourhood $\tilde{V}_{i}$ in $\mathcal{X}$ biholomorphic to a polydisk $\left\{\left(z_{1}, z_{2}\right) \in \mathbb{C}^{2}|| z_{1}\left|\leq \delta^{\prime},\right| z_{2} \mid \leq \delta^{\prime}\right\}$ on which $f$ takes the form $z_{1} z_{2}$.

If $\delta$ and $\delta^{\prime}$ are chosen correctly, then for $t$ sufficiently close to zero,

$$
\left\{\tilde{V}_{i} \cap \mathcal{X}_{t} \mid 1 \leq i \leq n\right\} \cup\left\{\tilde{U}_{i} \cap \mathcal{X}_{t} \mid 1 \leq i \leq n\right\}
$$

form an open cover of $\mathcal{X}_{t}$. Now each of the sets in this open cover can be written as $X_{\epsilon}(U)$ for some $U$ a one-dimensional (non-compact) affine manifold and $\epsilon=$ $-2 \pi / \ln |t|$. If $U$ is an open interval $(a, b) \subseteq \mathbb{R}$, then $X_{\epsilon}(U)$ is biholomorphic to the annulus

$$
\left\{z \in \mathbb{C}\left|e^{-2 \pi b / \epsilon} \leq\right| z \mid \leq e^{-2 \pi a / \epsilon}\right\}
$$

as $q=e^{2 \pi i(x+i y) / \epsilon}$ is a holomorphic coordinate on $X_{\epsilon}((a, b))$. Thus

$$
\tilde{U}_{i} \cap \mathcal{X}_{t} \cong X_{\epsilon}\left(\left(\frac{\epsilon \ln \delta}{2 \pi},-\frac{\epsilon \ln \delta}{2 \pi}\right)\right)
$$

with $\epsilon=-2 \pi / \ln |t|$. As $t \rightarrow 0$, the interval $(\epsilon \ln \delta / 2 \pi,-\epsilon \ln \delta / 2 \pi)$ shrinks to a point. So $\tilde{U}_{i} \cap \mathcal{X}_{t}$ is a smaller and smaller open subset of $\mathcal{X}_{t}$ as $t \rightarrow 0$ when we view things in this way. This argument suggests that every irreducible component should be associated to a point on $B$.

Now look at $\tilde{V}_{i} \cap \mathcal{X}_{t}$. This is

$$
\begin{aligned}
\left\{\left(z_{1}, z_{2}\right) \in \mathbb{C}^{2}|| z_{1}|,| z_{2} \mid<\delta^{\prime}, z_{1} z_{2}=t\right\} & \cong\left\{z \in \mathbb{C}|| t\left|/ \delta^{\prime} \leq\right| z \mid \leq \delta^{\prime}\right\} \\
& \cong X_{\epsilon}\left(\frac{-\epsilon}{2 \pi} \ln \delta^{\prime}, \frac{\epsilon}{2 \pi}\left(\ln \delta^{\prime}-\ln |t|\right)\right)
\end{aligned}
$$

with $\epsilon=-2 \pi / \ln |t|$. This interval approaches the unit interval $(0,1)$ as $t \rightarrow 0$. So the open set $\tilde{V}_{i} \cap \mathcal{X}_{t}$ ends up being a large portion of $\mathcal{X}_{t}$. We end up with $\mathcal{X}_{t}$, for small $t$, being a union of open sets of the form $X_{\epsilon}\left(\left(i+\epsilon^{\prime}, i+1-\epsilon^{\prime}\right)\right)$ (i.e. $\tilde{V}_{i} \cap \mathcal{X}_{\epsilon}$ ) and $X_{\epsilon}\left(\left(i-\epsilon^{\prime \prime}, i+\epsilon^{\prime \prime}\right)\right)$ (i.e. $\left.\tilde{U}_{i} \cap \mathcal{X}_{t}\right)$ for $\epsilon^{\prime}, \epsilon^{\prime \prime}$ sufficiently small. These should glue, at least approximately, to give $X_{\epsilon}(B)$. So we see that irreducible components of $\mathcal{X}_{0}$ seem to coincide with points on $B$, but intersections of components coincide with lines. In this way we see the dual intersection complex emerge.

Let us make one more observation before beginning with rigorous results in the next section. Suppose more generally we had a Gorenstein toroidal crossings degeneration of Calabi-Yau manifolds $f: \mathcal{X} \rightarrow D$. This means that every point $x \in \mathcal{X}$ has a neighbourhood isomorphic to an open set in an affine Gorenstein (i.e. the canonical class is a Cartier divisor) toric variety, with $f$ given locally by a monomial which vanishes exactly to order 1 on each codimension one toric stratum. This is a generalization of the notion of normal crossings, see [55. Very roughly, the above argument suggests that each irreducible component of the central fibre will correspond to a point of the Gromov-Hausdorff limit. The following exercise shows what kind of contribution to $B$ to expect from a point $x \in \mathcal{X}_{0}$ which is a zero-dimensional stratum in $\mathcal{X}_{0}$. 
EXERCISE 6.1. Suppose there is a point $x \in \mathcal{X}_{0}$ which has a neighbourhood isomorphic to a neighbourhood of a dimension zero torus orbit of an affine Gorenstein toric variety $Y_{x}$. Such an affine variety is specified as follows. Set $M=\mathbb{Z}^{n}$, $M_{\mathbb{R}}=M \otimes_{\mathbb{Z}} \mathbb{R}, N=\operatorname{Hom}_{\mathbb{Z}}(M, \mathbb{Z}), N_{\mathbb{R}}=N \otimes_{\mathbb{Z}} \mathbb{R}$ as in $\S 4$. Then there is a lattice polytope $\sigma \subseteq M_{\mathbb{R}}, n=\operatorname{dim} \mathcal{X}_{t}, C(\sigma):=\{(r m, r) \mid m \in \sigma, r \geq 0\} \subseteq M_{\mathbb{R}} \oplus \mathbb{R}$, $P:=C(\sigma)^{\vee} \cap(N \oplus \mathbb{Z})$ the monoid determined by the dual of the cone $C(\sigma)$, and finally, $Y_{x}=\operatorname{Spec} \mathbb{C}[P]$, and $f$ coincides with the monomial $z^{(0,1)}$.

Let us now take a small neighbourhood of $x$ of the form

$$
\tilde{U}_{\delta}=\left\{y \in \operatorname{Spec} \mathbb{C}[P]|| z^{p} \mid<\delta \text { for all } p \in P\right\} .
$$

This is an open set as the condition $\left|z^{p}\right|<\delta$ can be tested on a finite generating set for $P$, provided that $\delta<1$. Then show that for a given $t,|t|<1$ and $\epsilon=$ $-2 \pi / \log |t|$, if

$$
\sigma_{t}:=\left\{m \in M_{\mathbb{R}} \mid\langle p,(m, 1)\rangle>\frac{\log \delta}{\log |t|} \text { for all } p \in P\right\},
$$

then

$$
f^{-1}(t) \cap \tilde{U}_{\delta} \cong X_{\epsilon}\left(\sigma_{t}\right)
$$

Note that

$$
\sigma:=\left\{m \in M_{\mathbb{R}} \mid\langle p,(m, 1)\rangle \geq 0 \text { for all } p \in P\right\},
$$

so $\sigma_{t}$ is an open subset of $\sigma$, and as $t \rightarrow 0, \sigma_{t}$ converges to the interior of $\sigma$.

This observation will hopefully motivate the basic construction of the next section.

\section{Toric degenerations, the intersection complex and its dual}

We now return to rigorous statements. I would like to explain the basic ideas behind the program launched in $\mathbf{2 2}$. While I will use the previous sections as motivation, this work actually got its start when Siebert began a program of studying mirror symmetry via degenerations of Calabi-Yau manifolds. Work of Schröer and Siebert [54, [55] led Siebert to the idea that log structures on degenerations of Calabi-Yau manifolds would allow one to view mirror symmetry as an operation performed on degenerate Calabi-Yau varieties. Siebert observed that at a combinatorial level, mirror symmetry exchanged data pertaining to the log structure and a polarization. This will be explained more clearly in the following section, where we introduce log structures. Together, Siebert and I realised that the combinatorial data he was considering could be encoded naturally in the dual intersection complex of the degeneration, and that mirror symmetry then corresponded to a discrete Legendre transform on the dual intersection complex. It then became apparent that this approach provided an algebro-geometrization of the SYZ conjecture.

Here I will explain this program from the opposite direction, starting with the motivation of the previous section for introducing the dual intersection complex, and then work backwards until we arrive naturally at log structures. Much of the material in this section comes from [22], $\S 4$.

Definition 7.1. Let $f: \mathcal{X} \rightarrow D$ be a proper flat family of relative dimension $n$, where $D$ is a disk and $\mathcal{X}$ is a complex analytic space (not necessarily non-singular). We say $f$ is a toric degeneration of Calabi-Yau varieties if 
(1) $\mathcal{X}_{t}$ is an irreducible normal Calabi-Yau variety with only canonical singularities for $t \neq 0$. (The reader may like to assume $\mathcal{X}_{t}$ is smooth for $t \neq 0)$.

(2) If $\nu: \tilde{\mathcal{X}}_{0} \rightarrow \mathcal{X}_{0}$ is the normalization, then $\tilde{\mathcal{X}}_{0}$ is a disjoint union of toric varieties, the conductor locus $C \subseteq \tilde{\mathcal{X}}_{0}$ is reduced, and the map $C \rightarrow \nu(C)$ is unramified and generically two-to-one. (The conductor locus is a naturally defined scheme structure on the set where $\nu$ is not an isomorphism.) The square

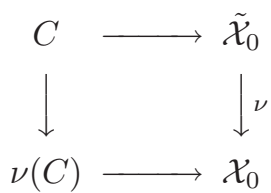

is cartesian and cocartesian.

(3) $\mathcal{X}_{0}$ is a reduced Gorenstein space and the conductor locus $C$ restricted to each irreducible component of $\tilde{\mathcal{X}}_{0}$ is the union of all toric Weil divisors of that component.

(4) There exists a closed subset $Z \subseteq \mathcal{X}$ of relative codimension $\geq 2$ such that $Z$ satisfies the following properties: $Z$ does not contain the image under $\nu$ of any toric stratum of $\tilde{\mathcal{X}}_{0}$, and for any point $x \in \mathcal{X} \backslash Z$, there is a neighbourhood $\tilde{U}_{x}$ (in the analytic topology) of $x$, an $n+1$-dimensional affine toric variety $Y_{x}$, a regular function $f_{x}$ on $Y_{x}$ given by a monomial, and a commutative diagram

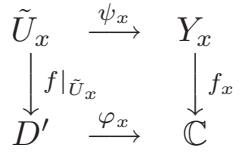

where $\psi_{x}$ and $\varphi_{x}$ are open embeddings and $D^{\prime} \subseteq D$. Furthermore, $f_{x}$ vanishes precisely once on each toric divisor of $Y_{x}$.

Example 7.2. Take $\mathcal{X}$ to be defined by the equation $t f_{4}+z_{0} z_{1} z_{2} z_{3}=0$ in $\mathbb{P}^{3} \times D$, where $D$ is a disk with coordinate $t$ and $f_{4}$ is a general homogeneous quartic polynomial on $\mathbb{P}^{3}$. It is easy to see that $\mathcal{X}$ is singular at the locus

$$
\left\{t=f_{4}=0\right\} \cap \operatorname{Sing}\left(\mathcal{X}_{0}\right) .
$$

As $\mathcal{X}_{0}$ is the coordinate tetrahedron, the singular locus of $\mathcal{X}_{0}$ consists of the six coordinate lines of $\mathbb{P}^{3}$, and $\mathcal{X}$ has four singular points along each such line, for a total of 24 singular points. Take $Z=\operatorname{Sing}(\mathcal{X})$. Then away from $Z$, the projection $\mathcal{X} \rightarrow D$ is normal crossings, which yields condition (4) of the definition of toric degeneration. It is easy to see all other conditions are satisfied.

EXAmple 7.3. Let $\Delta \subseteq M_{\mathbb{R}}$ be a reflexive polytope with dual $\nabla \subseteq N_{\mathbb{R}}$. Choose an integral strictly convex piecewise linear function $\breve{h}: M_{\mathbb{R}} \rightarrow \mathbb{R}$ as in $\S 4$. Consider the family in $\mathbb{P}_{\Delta} \times D$ defined by

$$
z^{0}+t \sum_{m \in \Delta \cap M} a_{m} t^{\check{h}^{\prime}(m)} z^{m}=0 .
$$

Here $z^{m}$ denotes the section of $\mathcal{O}_{\mathbb{P}_{\Delta}}(1)$ determined by the lattice point $m \in \Delta \cap M$ and $a_{m} \in k$ is a general choice of coefficient. So $z^{0}$ is the section which vanishes once on each toric boundary component of $\mathbb{P}_{\Delta}$. 
Now for most choices of $\check{h}$, this does not define a toric degeneration: the singularities are too bad. However, there is a natural toric variety in which to describe this degeneration. Set

$$
\tilde{\Delta}:=\left\{(m, l) \mid m \in \Delta, \quad l \geq \check{h}^{\prime}(m)\right\} \subseteq M_{\mathbb{R}} \oplus \mathbb{R} .
$$

The assumption that $\check{h}^{\prime}$ is convex implies $\tilde{\Delta}$ is convex, but of course is non-compact. Let $\tilde{\Sigma}$ be the normal fan to $\tilde{\Delta}$ in $N_{\mathbb{R}} \oplus \mathbb{R}$. Let $X(\tilde{\Sigma})$ denote the toric variety defined by the fan $\tilde{\Sigma}$. Then $\tilde{\Delta}$ is the Newton polytope of a line bundle $\mathcal{L}$ on $X(\tilde{\Sigma})$, and terms of the form $t^{\check{h}^{\prime}(m)} z^{m}$ can be interpreted as sections of this line bundle, corresponding to $\left(m, \check{h}^{\prime}(m)\right) \in \tilde{\Delta} \subseteq M_{\mathbb{R}} \oplus \mathbb{R}$. In addition, there is a natural map $X(\tilde{\Sigma}) \rightarrow \mathbb{A}^{1}$ defined by projection onto $\mathbb{R}$, and this map defines the regular function $t$. Thus (2) defines a hypersurface $\mathcal{X}_{\Delta} \subseteq X(\tilde{\Sigma})$ and $t$ defines a map $\mathcal{X}_{\Delta} \rightarrow \mathbb{A}^{1}$. This is a toric degeneration.

Without going into much detail, choosing a star decomposition of $\nabla$ as in $\S 4$ and a good polyhedral decomposition $\mathscr{P}$ of $\partial \nabla^{\breve{h}}$ is essentially the same as choosing a refinement $\tilde{\Sigma}^{\prime}$ of $\Sigma^{\prime}$ with particularly nice properties. This yields a partial resolution $X\left(\tilde{\Sigma}^{\prime}\right) \rightarrow X(\tilde{\Sigma})$. Then the proper transform of $\mathcal{X}_{\Delta}$ in $X\left(\tilde{\Sigma}^{\prime}\right), \mathcal{X}_{\Delta}^{\prime}$, yields another toric degeneration $\mathcal{X}_{\Delta}^{\prime} \rightarrow \mathbb{A}^{1}$. This is necessary to get a toric degeneration whose general fibre is a MPCP resolution of a hypersurface in a toric variety. For proofs, see [19], where the construction is generalized to complete intersections in toric varieties.

Given a toric degeneration $f: \mathcal{X} \rightarrow D$, we can build the dual intersection complex $(B, \mathscr{P})$ of $f$, as follows. Here $B$ is an integral affine manifold with singularities, and $\mathscr{P}$ is a polyhedral decomposition of $B$, a decomposition of $B$ into lattice polytopes. In fact, we will construct $B$ as a union of lattice polytopes. Specifically, let the normalisation of $\mathcal{X}_{0}, \tilde{\mathcal{X}}_{0}$, be written as a disjoint union $\amalg X_{i}$ of toric varieties $X_{i}, \nu: \tilde{\mathcal{X}}_{0} \rightarrow \mathcal{X}_{0}$ the normalisation. The strata of $\mathcal{X}_{0}$ are the elements of the set

$$
\operatorname{Strata}\left(\mathcal{X}_{0}\right)=\left\{\nu(S) \mid S \text { is a toric stratum of } X_{i} \text { for some } i\right\} .
$$

Here by toric stratum we mean the closure of a $\left(\mathbb{C}^{*}\right)^{n}$ orbit.

Let $\{x\} \in \operatorname{Strata}\left(\mathcal{X}_{0}\right)$ be a zero-dimensional stratum. Applying Definition 7.1 (4) to a neighbourhood of $x$, there is a toric variety $Y_{x}$ such that in a neighbourhood of $x, f: \mathcal{X} \rightarrow D$ is locally isomorphic to $f_{x}: Y_{x} \rightarrow \mathbb{C}$, where $f_{x}$ is given by a monomial. Now the condition that $f_{x}$ vanishes precisely once along each toric divisor of $Y_{x}$ is the statement that $Y_{x}$ is Gorenstein, and as such, it arises as in Exercise 6.1. Indeed, let $M, N$ be as usual, with $\operatorname{rank} M=\operatorname{dim} \mathcal{X}_{0}$. Then there is a lattice polytope $\sigma_{x} \subseteq M_{\mathbb{R}}$ such that $C\left(\sigma_{x}\right)=\{(r m, r) \mid m \in \sigma, r \geq 0\}$ is the cone defining the toric variety $Y_{x}$. As we saw in Exercise 6.1, a small neighbourhood of $x$ in $\mathcal{X}$ should contribute a copy of $\sigma_{x}$ to $B$, which provides the motivation for our construction. We can now describe how to construct $B$ by gluing together the polytopes

$$
\left\{\sigma_{x} \mid\{x\} \in \operatorname{Strata}\left(\mathcal{X}_{0}\right)\right\} .
$$

We will do this in the case that every irreducible component of $\mathcal{X}_{0}$ is in fact itself normal so that $\nu: X_{i} \rightarrow \nu\left(X_{i}\right)$ is an isomorphism. The reader may be able to imagine the more general construction. With this normality assumption, there is a one-to-one inclusion reversing correspondence between faces of $\sigma_{x}$ and elements of $\operatorname{Strata}\left(\mathcal{X}_{0}\right)$ containing $x$. We can then identify faces of $\sigma_{x}$ and $\sigma_{x^{\prime}}$ if they correspond to the same strata of $\mathcal{X}_{0}$. Some argument is necessary to show that this 
identification can be done via an integral affine transformation, but again this is not difficult.

Making these identifications, one obtains $B$. One can then prove

Lemma 7.4. If $\mathcal{X}_{0}$ is complex $n$ dimensional, then $B$ is an real $n$ dimensional manifold.

See 22, Proposition 4.10 for a proof.

Now so far $B$ is just a topological manifold, constructed by gluing together lattice polytopes. Let

$$
\mathscr{P}=\left\{\sigma \subseteq B \mid \sigma \text { is a face of } \sigma_{x} \text { for some zero-dimensional stratum } x\right\} .
$$

There is a one-to-one inclusion reversing correspondence between strata of $\mathcal{X}_{0}$ and elements of $\mathscr{P}$.

It only remains to give $B$ an affine structure with singularities. Let $\operatorname{Bar}(\mathscr{P})$ be the first barycentric subdivision of $\mathscr{P}$, and let $\Gamma$ be the union of simplices in $\operatorname{Bar}(\mathscr{P})$ not containing a vertex of $\mathscr{P}$ or intersecting the interior of a maximal cell of $\mathscr{P}$. If we then set $B_{0}:=B \backslash \Gamma$, we can define an affine structure on $B_{0}$ as follows. $B_{0}$ has an open cover

$$
\left\{W_{\sigma} \mid \sigma \in \mathscr{P} \text { maximal }\right\} \cup\left\{W_{v} \mid v \in \mathscr{P} \text { a vertex }\right\}
$$

where $W_{\sigma}=\operatorname{Int}(\sigma)$, the interior of $\sigma$, and

$$
W_{v}=\bigcup_{\substack{\tau \in \operatorname{Bar}(\mathscr{P}) \\ v \in \tau}} \operatorname{Int}(\tau)
$$

is the (open) star of $v$ in $\operatorname{Bar}(\mathscr{P})$, just as in $\S 4$.

We now define charts. As a maximal cell of $\mathscr{P}$ is of the form $\sigma_{x} \subseteq M_{\mathbb{R}}$, this inclusion induces a natural affine chart $\psi_{\sigma}: \operatorname{Int}(\sigma) \rightarrow M_{\mathbb{R}}$. On the other hand, a vertex $v$ of $\mathscr{P}$ corresponds to a codimension 0 stratum of $\mathcal{X}_{0}$, i.e. to an irreducible component $X_{i}$ for some $i$. Because this is a compact toric variety, it is defined by a complete fan $\Sigma_{i}$ in $M_{\mathbb{R}}$. Furthermore, there is a one-to-one correspondence between $p$-dimensional cones of $\Sigma_{i}$ and $p$-dimensional cells of $\mathscr{P}$ containing $v$ as a vertex, as they both correspond to strata of $\mathcal{X}_{0}$ contained in $X_{i}$. There is then a continuous map

$$
\psi_{v}: W_{v} \rightarrow M_{\mathbb{R}}
$$

which takes $W_{v} \cap \sigma$, for any $\sigma \in \mathscr{P}$ containing $v$ as a vertex, into the corresponding cone of $\Sigma_{i}$ integral affine linearly. Such a map is uniquely determined by the combinatorial correspondence and the requirement that it be integral affine linear on each cell. It is then obvious these charts define an integral affine structure on $B_{0}$. Thus we have constructed $(B, \mathscr{P})$.

EXAmPle 7.5. Let $f: \mathcal{X} \rightarrow D$ be a degeneration of elliptic curves to an $I_{n}$ fibre. Then $B$ is the circle $\mathbb{R} / n \mathbb{Z}$, decomposed by $\mathscr{P}$ into $n$ line segments of length one.

Example 7.6. Continuing with Example 7.3, the dual intersection complex constructed from the toric degenerations $\mathcal{X}_{\Delta}^{\prime} \rightarrow \mathbb{A}^{1}$ is the affine manifold with singularities structure with polyhedral decomposition $(B, \mathscr{P})$ constructed on $B=$ $\partial \nabla^{\check{h}}$ in $\S 4$. This is not particularly difficult to show. Again, for the proof and more general complete intersection case, see [19. 
Is the dual intersection complex the right affine manifold with singularities? The following theorem provides evidence for this, and gives the connection between this construction and the SYZ conjecture.

TheOREM 7.7. Let $\mathcal{X} \rightarrow D$ be a toric degeneration, with dual intersection complex $(B, \mathscr{P})$. Then there is an open set $U \subseteq B$ such that $B \backslash U$ retracts onto the discriminant locus $\Gamma$ of $B$, such that $\mathcal{X}_{t}$ contains an open subset $\mathscr{U}_{t}$ which is isomorphic as complex manifolds to a small deformation of a twist of $X_{\epsilon}(U)$, where $\epsilon=O(-1 / \ln |t|)$.

We will not be precise here about what we mean by small deformation; by twist, we mean a twist of the complex structure of $X_{\epsilon}(U)$ by a $B$-field. See 20 for a much more precise statement; the above statement is meant to give a feel for what is true. The proof, along with much more precise statements, will eventually appear in [21.

If $\mathcal{X} \rightarrow D$ is a polarized toric degeneration, i.e. if there is a relatively ample line bundle $\mathcal{L}$ on $\mathcal{X}$, then we can construct another affine manifold with singularities and polyhedral decomposition $(\check{B}, \check{\mathscr{P}})$, which we call the intersection complex, as follows.

For each irreducible component $X_{i}$ of $\mathcal{X}_{0},\left.\mathcal{L}\right|_{X_{i}}$ is an ample line bundle on a toric variety. Let $\sigma_{i} \subseteq N_{\mathbb{R}}$ denote the Newton polytope of this line bundle. There is then a one-to-one inclusion preserving correspondence between strata of $\mathcal{X}_{0}$ contained in $X_{i}$ and faces of $\sigma_{i}$. We can then glue together the $\sigma_{i}$ 's in the obvious way: if $Y$ is a codimension one stratum of $\mathcal{X}_{0}$, it is contained in two irreducible components $X_{i}$ and $X_{j}$, and defines faces of $\sigma_{i}$ and $\sigma_{j}$. These faces are affine isomorphic because they are both the Newton polytope of $\left.\mathcal{L}\right|_{Y}$, and we can then identify them in the canonical way. Thus we obtain a topological space $\check{B}$ with a polyhedral decomposition $\check{\mathscr{P}}$. We give it an affine structure with singularities in a similar manner as before. Again, let $\Gamma$ be the union of simplices in $\operatorname{Bar}(\check{\mathscr{P}})$ not containing a vertex of $\check{\mathscr{P}}$ or intersecting the interior of a maximal cell of $\check{\mathscr{P}}$. Setting $\check{B}_{0}:=\check{B} \backslash \Gamma$, this again has an open cover

$$
\left\{W_{\sigma} \mid \sigma \in \check{\mathscr{P}} \text { maximal }\right\} \cup\left\{W_{v} \mid v \in \check{\mathscr{P}} \text { a vertex }\right\} \text {. }
$$

As usual, as $W_{\sigma}$ is the interior of $\sigma$, it comes along with a canonical affine structure. On the other hand, a vertex $v$ of $\check{\mathscr{P}}$ corresponds to a dimension zero stratum $x$ of $\mathcal{X}_{0}$, and associated to $x$ is the polytope $\sigma_{x} \subseteq M_{\mathbb{R}}$. Let $\check{\Sigma}_{x}$ be the normal fan to $\sigma_{x}$ in $N_{\mathbb{R}}$. Then there is a one-to-one inclusion preserving correspondence between cones in $\check{\Sigma}_{x}$ and strata of $\mathcal{X}_{0}$ containing $x$. This correspondence allows us to define a chart

$$
\check{\psi}_{v}: W_{v} \rightarrow N_{\mathbb{R}}
$$

which takes $W_{v} \cap \check{\sigma}$, for any $\check{\sigma} \in \check{\mathscr{P}}$ containing $v$ as a vertex, into the corresponding cone of $\check{\Sigma}_{x}$ in an integral affine linear way. This gives the manifestly integral affine structure on $\check{B}_{0}$, and hence defines the intersection complex $(\check{B}, \check{\mathscr{P}})$.

Analogously to Theorem 7.7, we expect

Conjecture 7.8. Let $\mathcal{X} \rightarrow D$ be a polarized toric degeneration, with intersection complex $(\check{B}, \check{\mathscr{P}})$. Let $\omega_{t}$ be a Kähler form on $\mathcal{X}_{t}$ representing the first Chern class of the polarization. Then there is an open set $\check{U} \subseteq \check{B}$ such that $\check{B} \backslash \check{U}$ retracts onto the discriminant locus $\Gamma$ of $\check{B}$, such that $\mathcal{X}_{t}$ is a symplectic compactification of $\check{X}(\check{U})$ for any $t$. 
I don't expect this to be particularly difficult: it should be amenable to the techniques of Ruan [53, but has not been carried out.

The relationship between the intersection complex and the dual intersection complex can be made more precise by introducing multi-valued piecewise linear functions, in analogy with the multi-valued convex functions of Definition 2.3.

DeFinition 7.9. Let $B$ be an affine manifold with singularities with polyhedral decomposition $\mathscr{P}$. Then a multi-valued piecewise linear function $\varphi$ on $B$ is a collection of continuous functions on an open cover $\left\{\left(U_{i}, \varphi_{i}\right)\right\}$ such that $\varphi_{i}$ is affine linear on each cell of $\mathscr{P}$ intersecting $U_{i}$, and on $U_{i} \cap U_{j}, \varphi_{i}-\varphi_{j}$ is affine linear. Furthermore, for any $\sigma \in \mathscr{P}$, in a neighbourhood of each point $x \in U_{i} \cap \operatorname{Int}(\sigma)$, there is an affine linear function $\psi$ such that $\varphi_{i}-\psi$ is zero on $\sigma$.

To explain this last condition, and to clarify additional structure on $B$, let us examine a property of the polyhedral decomposition $\mathscr{P}$ of $B$ when $(B, \mathscr{P})$ is a dual intersection complex.

Consider any $p$-dimensional cell $\sigma \in \mathscr{P}$. This corresponds to an $n$-p-dimensional stratum $X_{\sigma} \subseteq \mathcal{X}_{0}$, and as such, it is a toric variety defined by a fan $\Sigma_{\sigma}$ in $\mathbb{R}^{n-p}$. Now for any vertex $v$ of $\sigma, X_{\sigma}$ is a toric stratum of the irreducible component $X_{v}$ of $\mathcal{X}_{0}$. Thus $\Sigma_{\sigma}$ can be obtained as a quotient fan of $\Sigma_{v}$. In other words, there is a p-dimensional cone $K_{\sigma}$ of $\Sigma_{v}$ corresponding to $\sigma$ such that

$$
\Sigma_{\sigma}=\Sigma_{v}\left(K_{\sigma}\right):=\left\{\left(K+\mathbb{R} K_{\sigma}\right) / \mathbb{R} K_{\sigma} \mid K \in \Sigma_{v}, K \supseteq K_{\sigma}\right\} .
$$

In particular, there is an open neighbourhood $U_{v, \sigma}$ of $\operatorname{Int}\left(K_{\sigma}\right)$ and an integral linear map $S_{v, \sigma}: U_{v, \sigma} \rightarrow \mathbb{R}^{n-p}$ such that

$$
\left\{S_{v, \sigma}^{-1}(K) \mid K \in \Sigma_{\sigma}\right\}=\left\{U_{v, \sigma} \cap K \mid K \in \Sigma_{v}, K \supseteq K_{\sigma}\right\} .
$$

Let $U_{\sigma}$ be a small open neighbourhood of $\operatorname{Int}(\sigma)$ in $B$; if taken sufficiently small the maps $S_{v, \sigma}$ can be viewed as being defined on open subsets of $U_{\sigma}$ and patch to give an integral affine submersion $S_{\sigma}: U_{\sigma} \rightarrow \mathbb{R}^{n-p}$, where $U_{\sigma}=\bigcup_{v \in \sigma} U_{v, \sigma}$ is an open neighbourhood of $\operatorname{Int}(\sigma)$. This map has the property that

$$
\left\{S_{\sigma}^{-1}(K) \mid K \in \Sigma_{\sigma}\right\}=\left\{U_{\sigma} \cap \tau \mid \tau \supseteq \sigma, \tau \in \mathscr{P}\right\} .
$$

In general we call a polyhedral decomposition toric if for all $\sigma \in \mathscr{P}$ there is always such an integral affine linear map $S_{\sigma}: U_{\sigma} \rightarrow \mathbb{R}^{n-p}$ from a neighbourhood $U_{\sigma}$ of $\operatorname{Int}(\sigma)$ and a fan $\Sigma_{\sigma}$ in $\mathbb{R}^{p}$ with the above property. (See [22, Definition 1.22 for a perhaps too precise definition of toric polyhedral decompositions. The definition there is complicated by allowing cells to be self-intersecting, or equivalently, allowing irreducible components of $\mathcal{X}_{0}$ to be non-normal.) We can think of the fan $\Sigma_{\sigma}$ as being the fan structure of $\mathscr{P}$ transverse to $\sigma$ at a point in the interior of $\sigma$. The main point for a dual intersection complex is that this fan structure is determined by $X_{\sigma}$, and this is independent of the choice of the point in the interior of $\sigma$.

Now let us return to piecewise linear functions. Suppose we are given a polarized toric degeneration $\mathcal{X} \rightarrow D$. We in fact obtain a piecewise linear function $\varphi$ on the dual intersection complex $(B, \mathscr{P})$ as follows. Restricting to any toric stratum $X_{\sigma},\left.\mathcal{L}\right|_{X_{\sigma}}$ is determined completely by an integral piecewise linear function $\bar{\varphi}_{\sigma}$ on $\Sigma_{\sigma}$, well-defined up to a choice of linear function. Pulling back this piecewise linear function via $S_{\sigma}$ to $U_{\sigma}$, we obtain a collection of piecewise linear functions $\left\{\left(U_{\sigma}, \varphi_{\sigma}\right) \mid \sigma \in \mathscr{P}\right\}$. The fact that $\left.\left(\left.\mathcal{L}\right|_{X_{\tau}}\right)\right|_{X_{\sigma}}=\left.\mathcal{L}\right|_{X_{\sigma}}$ for $\tau \subseteq \sigma$ implies 
that on overlaps $\varphi_{\sigma}$ and $\varphi_{\tau}$ differ by at most a linear function. So $\left\{\left(U_{\sigma}, \varphi_{\sigma}\right)\right\}$ defines a multi-valued piecewise linear function. The last condition in the definition of multi-valued piecewise linear function then reflects the need for the function to be locally a pull-back of a function via $S_{\sigma}$ in a neighbourhood of $\sigma$. In fact, given any multi-valued piecewise linear function $\varphi$ on $(B, \mathscr{P})$ with $\mathscr{P}$ a toric polyhedral decomposition of $B, \varphi$ is determined by functions $\bar{\varphi}_{\sigma}$ on $\Sigma_{\sigma}$ for $\sigma \in \mathscr{P}$, via pull-back by $S_{\sigma}$.

If $\mathcal{L}$ is ample, then the piecewise linear function determined by $\left.\mathcal{L}\right|_{X_{\sigma}}$ is strictly convex. So we say a multi-valued piecewise linear function is strictly convex if $\bar{\varphi}_{\sigma}$ is strictly convex for each $\sigma \in \mathscr{P}$.

Now suppose we are given abstractly a triple $(B, \mathscr{P}, \varphi)$ with $B$ an integral affine manifold with singularities with a toric polyhedral decomposition $\mathscr{P}$, and $\varphi$ a strictly convex multi-valued piecewise linear function on $B$. Then we construct the discrete Legendre transform $(\check{B}, \check{\mathscr{P}}, \check{\varphi})$ of $(B, \mathscr{P}, \varphi)$ as follows.

$\check{B}$ will be constructed by gluing together Newton polytopes. If we view, for $v$ a vertex of $\mathscr{P}$, the fan $\Sigma_{v}$ as living in $M_{\mathbb{R}}$, then the Newton polytope of $\bar{\varphi}_{v}$ is

$$
\check{v}=\left\{x \in N_{\mathbb{R}} \mid\langle x, y\rangle \geq-\bar{\varphi}_{v}(y) \quad \forall y \in M_{\mathbb{R}}\right\} .
$$

There is a one-to-one order reversing correspondence between faces of $\check{v}$ and cells of $\mathscr{P}$ containing $v$. Furthermore, if $\sigma$ is the smallest cell of $\mathscr{P}$ containing two vertices $v$ and $v^{\prime}$, then the corresponding faces of $\check{v}$ and $\check{v}^{\prime}$ are integral affine isomorphic, as they are both isomorphic to the Newton polytope of $\bar{\varphi}_{\sigma}$. Thus we can glue $\check{v}$ and $\check{v}^{\prime}$ along this common face. After making all these identifications, we obtain a cell complex $(\check{B}, \check{\mathscr{P}})$, which is really just the dual cell complex of $(B, \mathscr{P})$. Of course, we have some additional information, namely an affine structure on the interior of each maximal cell of $\check{\mathscr{P}}$. To give $\check{B}$ an integral affine structure with singularities, one proceeds as usual, using this affine structure along with an identification of a neighbourhood of each vertex of $\check{\mathscr{P}}$ with the normal fan of the corresponding maximal cell of $\mathscr{P}$.

Finally, the function $\varphi$ has a discrete Legendre transform $\check{\varphi}$ on $(\check{B}, \check{\mathscr{P}})$. We have no choice but to define $\check{\varphi}$ in a neighbourhood of a vertex $\check{\sigma} \in \check{\mathscr{P}}$ dual to a maximal cell $\sigma \in \mathscr{P}$ to be a piecewise linear function whose Newton polytope is $\sigma$, i.e.

$$
\bar{\varphi}_{\check{\sigma}}(y)=-\inf \left\{\langle y, x\rangle \mid x \in \sigma \subseteq M_{\mathbb{R}}\right\}
$$

This gives $(\check{B}, \check{\mathscr{P}}, \check{\varphi})$, the discrete Legendre transform of $(B, \mathscr{P}, \varphi)$. If $B$ is $\mathbb{R}^{n}$, then this coincides with the classical notion of a discrete Legendre transform. The discrete Legendre transform has several relevant properties:

- The discrete Legendre transform of $(\check{B}, \check{\mathscr{P}}, \check{\varphi})$ is $(B, \mathscr{P}, \varphi)$.

- If we view the underlying topological spaces $B$ and $\check{B}$ as being identified by being the underlying space of dual cell complexes, then $\Lambda_{B_{0}} \cong \check{\Lambda}_{\check{B}_{0}}$ and $\check{\Lambda}_{B_{0}} \cong \Lambda_{\check{B}_{0}}$, where the subscript denotes which affine structure is being used to define $\Lambda$ or $\check{\Lambda}$.

This hopefully makes it clear that the discrete Legendre transform is a suitable replacement for the duality provided to us by the Legendre transform of $\S 2$.

Finally, it leads to what we may think of as an algebro-geometric SYZ procedure. In analogy with the procedure suggested in $\$ 5$, we follow these steps: 
(1) We begin with a toric degeneration of Calabi-Yau manifolds $\mathcal{X} \rightarrow D$ with an ample polarization.

(2) Construct $(B, \mathscr{P}, \varphi)$ from this data, as explained above.

(3) Perform the discrete Legendre transform to obtain $(\check{B}, \check{\mathscr{P}}, \check{\varphi})$.

(4) Try to construct a polarized degeneration of Calabi-Yau manifolds $\check{\mathcal{X}} \rightarrow D$ whose dual intersection complex is $(\check{B}, \check{\mathscr{P}}, \check{\varphi})$.

EXAMPLE 7.10. The discrete Legendre transform enables us to reproduce Batyrev duality. Returning to the construction of $\S 4$ and Example 7.3 , choosing a strictly convex piecewise linear function on $\tilde{\Sigma}^{\prime}$ corresponding to a line bundle $\mathcal{L}$ induces a polarization of $\mathcal{X}_{\Delta}^{\prime}$. This then gives us a strictly convex multi-valued piecewise linear function $\varphi_{\mathcal{L}}$ on $(B, \mathscr{P})$, hence a discrete Legendre transform $\left(\check{B}, \check{\mathscr{P}}, \check{\varphi}_{\mathcal{L}}\right)$. In 19] I showed that this is the dual intersection complex associated to some choice of subdivision $\widetilde{\Sigma^{\prime}}$ of $\widetilde{\Sigma}$ obtained by interchanging the roles of $\nabla$ and $\Delta$ in the construction of $\S 4$. As an exercise, you can check the following for yourself. If we take $\breve{h}=\check{\varphi}$, and in addition define

$$
h=\varphi: N_{\mathbb{R}} \rightarrow \mathbb{R}
$$

to take the value 1 on the primitive generator of each one-dimensional cone on $\Sigma$, the normal fan to $\Delta$, then from $\S 4$ we obtain an affine structure with singularities on $B=\partial \nabla$, and completely symetrically using $h$ we also obtain such a structure on $\check{B}=\partial \Delta$. These manifolds come with polyhedral decomposition $\mathscr{P}$ and $\check{\mathscr{P}}$ consisting of all proper faces of $\nabla$ and $\Delta$ respectively. The anti-canonical polarizations on $\mathbb{P}_{\Delta}$ and $\mathbb{P}_{\nabla}$ induce multi-valued piecewise linear functions $\psi, \check{\psi}$ on $B$ and $\check{B}$ respectively. Then show $(B, \mathscr{P}, \psi)$ and $(\check{B}, \check{\mathscr{P}}, \breve{\psi})$ are discrete Legendre transforms of each other.

Thus Batyrev (and Batyrev-Borisov) duality is a special case of this construction.

The only step missing in this mirror symmetry algorithm is the last:

QueSTION 7.11 (The reconstruction problem, Version II). Given $(B, \mathscr{P}, \varphi)$, is it possible to construct a polarized toric degeneration $\mathcal{X} \rightarrow D$ whose dual intersection complex is $(B, \mathscr{P}, \varphi)$ ?

It is fairly obvious how to reconstruct the central fibre $\mathcal{X}_{0}$ of a degeneration from the data $(\check{B}, \check{\mathscr{P}}, \check{\varphi})$, and we will see this explicitly in $\S 8$. One could naively hope that this reducible variety has good deformation theory and it can be smoothed. However, in general its deformation theory is ill-behaved.

As initially observed in the normal crossings case by Kawamata and Namikawa in [36, one needs to put some additional structure on $\mathcal{X}_{0}$ before it has good deformation theory. This structure is a log structure, and introducing log structures allows us to study many aspects of mirror symmetry directly on the degenerate fibre itself.

We shall do this in the next section, but first, let me address the question of how general this mirror symmetry construction might be:

ConjeCture 7.12. If $f: \mathcal{X} \rightarrow D$ is a large complex structure limit degeneration, then $f$ is birationally equivalent to a toric degeneration $f^{\prime}: \mathcal{X}^{\prime} \rightarrow D$.

The condition of being a large complex structure limit, as defined by Morrison in 47, is a stronger one than maximally unipotent. Why should I imagine 
something like this to be true? Well, fantasizing freely, we would expect that after choosing a polarization and Ricci-flat metric on fibres $\mathcal{X}_{t}$, we have a sequence $\left(\mathcal{X}_{t}, g_{t}\right)$ converging to $B$ an affine manifold with singularities. Now in general an affine manifold with singularities need not arise as the dual intersection complex of a toric degeneration, first of all because it need not have a toric polyhedral decomposition. For example, even in two dimensions there are orbifold singularities (corresponding to singular elliptic fibres which are not semi-stable) which do not arise in dual intersection complexes of toric degenerations, yet can occur as the base of a special Lagrangian fibration on a K3 surface. However, the general base does arise as the dual intersection complex of a toric degeneration in the K3 case. The hope is that the condition of large complex structure limit forces the singularities of $B$ to be "sufficiently general" so that one can construct a nice toric polyhedral decomposition $\mathscr{P}$ on $B$, and from this construct a toric degeneration. Presumably, this toric degeneration will be, if picked correctly, birational to the original one.

This argument of course is rather hand-wavy, but I believe it provides some moral expectation that there might be a large class of degenerations for which our method applies. I note that one can prove the conjecture in the case of K3 surfaces.

We now come to the technical heart of the program laid out in $\mathbf{2 2}$. Some aspects of this program are quite technical, so the goal here is to explain the highlights of [22] as simply possible.

\section{Log structures}

We first introduce the log structures of Fontaine-Illusie and Kato ([32, 35]).

Definition 8.1. A log structure on a scheme (or analytic space) $X$ is a (unital) homomorphism

$$
\alpha_{X}: \mathcal{M}_{X} \rightarrow \mathcal{O}_{X}
$$

of sheaves of (multiplicative and commutative) monoids inducing an isomorphism $\alpha_{X}^{-1}\left(\mathcal{O}_{X}^{\times}\right) \rightarrow \mathcal{O}_{X}^{\times}$. The triple $\left(X, \mathcal{M}_{X}, \alpha_{X}\right)$ is then called a $\log$ space. We often write the whole package as $X^{\dagger}$.

A morphism of $\log$ spaces $F: X^{\dagger} \rightarrow Y^{\dagger}$ consists of a morphism $\underline{F}: X \rightarrow$ $Y$ of underlying spaces together with a homomorphism $F^{\#}: \underline{F}^{-1}\left(\mathcal{M}_{Y}\right) \rightarrow \mathcal{M}_{X}$ commuting with the structure homomorphisms:

$$
\alpha_{X} \circ F^{\#}=\underline{F}^{*} \circ \alpha_{Y} .
$$

The key examples:

ExAmples 8.2. (1) Let $X$ be a scheme and $Y \subseteq X$ a closed subset of codimension one. Denote by $j: X \backslash Y \rightarrow X$ the inclusion. Then the inclusion

$$
\alpha_{X}: \mathcal{M}_{X}=j_{*}\left(\mathcal{O}_{X \backslash Y}^{\times}\right) \cap \mathcal{O}_{X} \rightarrow \mathcal{O}_{X}
$$

of the sheaf of regular functions with zeroes contained in $Y$ is a $\log$ structure on $X$. This is called a divisorial log structure on $X$.

(2) A prelog structure, i.e. an arbitrary homomorphism of sheaves of monoids $\varphi: \mathcal{P} \rightarrow \mathcal{O}_{X}$, defines an associated $\log$ structure $\mathcal{M}_{X}$ by

$$
\mathcal{M}_{X}=\left(\mathcal{P} \oplus \mathcal{O}_{X}^{\times}\right) /\left\{\left(p, \varphi(p)^{-1}\right) \mid p \in \varphi^{-1}\left(\mathcal{O}_{X}^{\times}\right)\right\}
$$

and $\alpha_{X}(p, h)=h \cdot \varphi(p)$. 
(3) If $f: X \rightarrow Y$ is a morphism of schemes and $\alpha_{Y}: \mathcal{M}_{Y} \rightarrow \mathcal{O}_{Y}$ is a $\log$ structure on $Y$, then the prelog structure $f^{-1}\left(\mathcal{M}_{Y}\right) \rightarrow \mathcal{O}_{X}$ defines an associated $\log$ structure on $X$, the pull-back log structure.

(4) In (1) we can pull back the log structure on $X$ to $Y$ using (3). Thus in particular, if $\mathcal{X} \rightarrow D$ is a toric degeneration, the inclusion $\mathcal{X}_{0} \subseteq \mathcal{X}$ gives a $\log$ structure on $\mathcal{X}$ and an induced $\log$ structure on $\mathcal{X}_{0}$. Similarly the inclusion $0 \in D$ gives a $\log$ structure on $D$ and an induced one on 0 . Here $\mathcal{M}_{0}=\mathbb{C}^{\times} \oplus \mathbb{N}$, where $\mathbb{N}$ is the (additive) monoid of natural (non-negative) numbers, and

$$
\alpha_{0}(h, n)= \begin{cases}h & n=0 \\ 0 & n \neq 0\end{cases}
$$

$0^{\dagger}$ is usually called the standard log point.

We then have $\log$ morphisms $\mathcal{X}^{\dagger} \rightarrow D^{\dagger}$ and $\mathcal{X}_{0}^{\dagger} \rightarrow 0^{\dagger}$.

(5) If $\sigma \subseteq M_{\mathbb{R}}=\mathbb{R}^{n}$ is a convex rational polyhedral cone, $\sigma^{\vee} \subseteq N_{\mathbb{R}}$ the dual cone, let $P=\sigma^{\vee} \cap N$ : this is a monoid. The affine toric variety defined by $\sigma$ can be written as $X=\operatorname{Spec} \mathbb{C}[P]$. We then have a pre-log structure induced by the homomorphism of monoids

$$
P \rightarrow \mathbb{C}[P]
$$

given by $p \mapsto z^{p}$. There is then an associated log structure on $X$. This is in fact the same as the log structure induced by $\partial X \subseteq X$, where $\partial X$ is the toric boundary of $X$, i.e. the union of toric divisors of $X$.

If $p \in P$, then the monomial $z^{p}$ defines a map $f: X \rightarrow \operatorname{Spec} \mathbb{C}[\mathbb{N}] \quad(=\operatorname{Spec} \mathbb{C}[t])$ which is a $\log$ morphism with the $\log$ structure on Spec $\mathbb{C}[\mathbb{N}]$ induced similarly by $\mathbb{N} \rightarrow \mathbb{C}[\mathbb{N}]$. The fibre $X_{0}=\operatorname{Spec} \mathbb{C}[P] /\left(z^{p}\right)$ is a subscheme of $X$, and there is an induced $\log$ structure on $X_{0}$, and a map $X_{0}^{\dagger} \rightarrow 0^{\dagger}$ as in (4). $f$ is an example of a $\log$ smooth morphism. Essentially all $\log$ smooth morphisms are étale locally of this form (if $\mathbb{N}$ is replaced by a more general monoid). See [34 for details.

Condition (4) of Definition 7.1 in fact implies that locally, away from $Z, \mathcal{X}^{\dagger}$ and $\mathcal{X}_{0}^{\dagger}$ are of the above form. So we should view $\mathcal{X}^{\dagger} \rightarrow D^{\dagger}$ as $\log$ smooth away from $Z$, and from the log point of view, $\mathcal{X}_{0}^{\dagger}$ can be treated much like a non-singular scheme away from $X$. We will see this explicitly below when we talk about differentials.

On a log scheme $X^{\dagger}$ there is always an exact sequence

$$
1 \longrightarrow \mathcal{O}_{X}^{\times} \stackrel{\alpha^{-1}}{\longrightarrow} \mathcal{M}_{X} \longrightarrow \overline{\mathcal{M}}_{X} \longrightarrow 0
$$

where we write the quotient sheaf of monoids $\overline{\mathcal{M}}_{X}$ additively. We call $\overline{\mathcal{M}}_{X}$ the ghost sheaf of the log structure. I like to view $\overline{\mathcal{M}}_{X}$ as specifying the combinatorial information associated to the log structure. For example, if $X^{\dagger}$ is induced by the Cartier divisor $Y \subseteq X$ with $X$ normal, then the stalk $\overline{\mathcal{M}}_{X, x}$ at $x \in X$ is the monoid of effective Cartier divisors on a neighbourhood of $x$ supported on $Y$.

ExERCISE 8.3. Show that in Example 8.2. (5), $\overline{\mathcal{M}}_{X, x}=P$ if $\operatorname{dim} \sigma=n$ and $x$ is the unique zero-dimensional torus orbit of $X$. More generally,

$$
\overline{\mathcal{M}}_{X, x}=\frac{\tau^{\vee} \cap N}{\tau^{\perp} \cap N}=\operatorname{Hom}_{\text {monoid }}(\tau \cap M, \mathbb{N}),
$$

when $x \in X$ is in the torus orbit corresponding to a face $\tau$ of $\sigma$. In particular, $\tau$ can be recovered as $\operatorname{Hom}_{\text {monoid }}\left(\overline{\mathcal{M}}_{X, x}, \mathbb{R}_{\geq 0}^{+}\right)$, where $\mathbb{R}_{\geq 0}^{+}$is the additive monoid of non-negative real numbers. 
Another important fact for us is that if $f: Y \rightarrow X$ is a morphism with $X$ carrying a $\log$ structure, and $Y$ is given the pull-back $\log$ structure, then $\overline{\mathcal{M}}_{Y}=f^{-1} \overline{\mathcal{M}}_{X}$. In the case that $\mathcal{M}_{X}$ is induced by an inclusion of $Y \subseteq X, \overline{\mathcal{M}}_{X}$ is supported on $Y$, so we can equate $\overline{\mathcal{M}}_{X}$ and $\overline{\mathcal{M}}_{Y}$, the ghost sheaves for the divisorial log structure on $X$ and its restriction to $Y$. Putting this together with Exercise 8.3 and the definition of dual intersection complex, we see that given a toric degeneration $\mathcal{X} \rightarrow D$ the dual intersection complex completely determines the ghost sheaf $\overline{\mathcal{M}}_{\mathcal{X}}=\overline{\mathcal{M}}_{\mathcal{X}_{0}}$ off of $Z$. We in fact take the view that anyway the $\log$ structure on $Z$ is not particularly well-behaved, and we always ignore it on $Z$. In fact, given a $\log$ structure $\mathcal{M}_{\mathcal{X}_{0} \backslash Z}$ on $\mathcal{X}_{0} \backslash Z$, this defines a push-forward $\log$ structure $\mathcal{M}_{\mathcal{X}_{0}}:=j_{*} \mathcal{M}_{\mathcal{X}_{0} \backslash Z}$. There is an induced map $\alpha: \mathcal{M}_{\mathcal{X}_{0}} \rightarrow \mathcal{O}_{\mathcal{X}_{0}}$, as $j_{*} \mathcal{O}_{\mathcal{X}_{0} \backslash Z}=\mathcal{O}_{\mathcal{X}_{0}}$ because $\mathcal{X}_{0}$ is Cohen-Macaulay. Thus in what follows, if we have determined a $\log$ structure on $\mathcal{X}_{0} \backslash Z$, we just as well get a $\log$ structure on $\mathcal{X}_{0}$ and will not concern ourselves with the behaviour of this $\log$ structure along $Z$.

All this gives the necessary hint for working backwards, to go from $(B, \mathscr{P})$ to $\mathcal{X}_{0}^{\dagger}$. Suppose we are given an integral affine manifold with singularities $B$ with toric polyhedral decomposition $\mathscr{P}$. At each vertex $v$ of $\mathscr{P}, \mathscr{P}$ locally looks like a fan $\Sigma_{v}$, defining a toric variety $X_{v}$. For every edge $\omega \in \mathscr{P}$ with endpoints $v$ and $w, \omega$ defines a ray in both fans $\Sigma_{v}$ and $\Sigma_{w}$, hence toric divisors $D_{\omega}^{v} \subseteq X_{v}$, $D_{\omega}^{w} \subseteq X_{w}$. The condition that $\mathscr{P}$ is a toric polyhedral decomposition tells us that $D_{\omega}^{v}$ and $D_{\omega}^{w}$ are isomorphic toric varieties, and we can choose a torus equivariant isomorphism $s_{\omega}: D_{\omega}^{v} \rightarrow D_{\omega}^{w}$ for each edge $\omega$. If we choose these gluing maps to satisfy a certain compatibility condition on codimension two strata (we leave it to the reader to write down this simple compatibility condition), then we can glue together the $X_{v}$ 's to obtain, in general, an algebraic space we write as $X_{0}(B, \mathscr{P}, s)$, where $s=\left(s_{\omega}\right)$ is the collection of gluing maps. (In [22], we describe the gluing data in a slightly different, but equivalent, way). We call $s$ closed gluing data. This is how we construct a potential central fibre of a toric degeneration.

Now $X_{0}(B, \mathscr{P}, s)$ cannot be a central fibre of a toric degeneration unless it carries a log structure of the correct sort. There are many reasons this may not happen. First, if $s$ is poorly chosen, there may be zero-dimensional strata of $X_{0}(B, \mathscr{P}, s)$ which do not have neighbourhoods locally étale isomorphic to the toric boundary of an affine toric variety; this is a minimum prerequisite. As a result, we have to restrict attention to closed gluing data induced by what we call open gluing data. Explicitly, each maximal cell $\sigma \in \mathscr{P}$ defines an affine toric variety $U(\sigma)$ given by the cone $C(\sigma) \subseteq M_{\mathbb{R}} \oplus \mathbb{R}$, assuming we view $\sigma \subseteq M_{\mathbb{R}}$ as a lattice polytope. Let $V(\sigma) \subseteq U(\sigma)$ be the toric boundary. It turns out, as we show in 22 , that a necessary condition for $X_{0}(B, \mathscr{P}, s)$ to be the central fibre of a toric degeneration is that it is obtained by dividing out $\coprod_{\sigma \in \mathscr{P}_{\max }} V(\sigma)$ by an étale equivalence relation. In other words, we are gluing together the $V(\sigma)$ 's to obtain an algebraic space, and those étale equivalence relations which produce algebraic spaces of the form $X_{0}(B, \mathscr{P}, s)$ are easily determined. This is carried out in detail in [22, $\S 2$. The construction there appears technically difficult because of the necessity of dealing with algebraic spaces, but is basically straightforward. The basic point is that if $\sigma_{1}, \sigma_{2} \in \mathscr{P}$ are two maximal cells, with $\sigma_{1} \cap \sigma_{2}=\tau$, then $\tau$ determines faces of the cones $C\left(\sigma_{1}\right)$ and $C\left(\sigma_{2}\right)$, hence open subsets $U_{i}(\tau) \subseteq U\left(\sigma_{i}\right)$, with toric boundaries $V_{i}(\tau) \subseteq V\left(\sigma_{i}\right)$. Now in general there is no natural isomorphism between $U_{1}(\tau)$ and 
$U_{2}(\tau)$ : this is a problem when $\sigma_{1} \cap \sigma_{2} \cap \Gamma \neq \emptyset$, where $\Gamma$ is as usual the singular locus of $B$. However, crucially $V_{1}(\tau)$ and $V_{2}(\tau)$ are naturally isomorphic, and we can choose compatible equivariant isomorphisms to obtain open gluing data. Choosing open gluing data allows us to define the étale equivalence relation: we are just gluing any two sets $V\left(\sigma_{1}\right), V\left(\sigma_{2}\right)$ via the chosen isomorphism between $V_{1}(\tau)$ and $V_{2}(\tau)$. Any choice of open gluing data $s$ gives rise in this way to an algebraic space $X_{0}(B, \mathscr{P}, s)$, and to any choice of open gluing data there is associated closed gluing data $s^{\prime}$ such that $X_{0}(B, \mathscr{P}, s) \cong X_{0}\left(B, \mathscr{P}, s^{\prime}\right)$.

The advantage of using open gluing data is that each $V(\sigma)$ for $\sigma \in \mathscr{P}_{\max }$ carries a $\log$ structure induced by the divisorial $\log$ structure $V(\sigma) \subseteq U(\sigma)$. Unfortunately, these $\log$ structures are not identified under the open gluing maps, precisely because of a lack of a natural isomorphism between the $U_{i}(\tau)$ 's cited above. However, the ghost sheaves of the log structures are isomorphic. So the ghost sheaves $\overline{\mathcal{M}}_{V(\sigma)}$ glue to give a ghost sheaf of monoids $\overline{\mathcal{M}}_{X_{0}(B, \mathscr{P}, s)}$. Summarizing what we have said so far: (this is a combination of results of $[\mathbf{2 2}], \S \S 2,4$ )

TheOREM 8.4. Given $(B, \mathscr{P})$, if $s$ is closed gluing data, and $\mathcal{X}_{0}=X_{0}(B, \mathscr{P}, s)$ is the central fibre of a toric degeneration $\mathcal{X} \rightarrow D$ with dual intersection complex $(B, \mathscr{P})$, then $s$ is induced by open gluing data and $\left.\left.\overline{\mathcal{M}}_{\mathcal{X}_{0}}\right|_{\mathcal{X}_{0} \backslash Z} \cong \overline{\mathcal{M}}_{X_{0}(B, \mathscr{P}, s)}\right|_{\mathcal{X}_{0} \backslash Z}$.

This is as far as we can get with the combinatorics. The next point is to attempt to construct $\mathcal{M}_{X_{0}(B, \mathscr{P}, s)}$. The idea is that $\mathcal{M}_{X_{0}(B, \mathscr{P}, s)}$ is an extension of $\overline{\mathcal{M}}_{X_{0}(B, \mathscr{P}, s)}$ by $\mathcal{O}_{X_{0}(B, \mathscr{P}, s)}^{\times}$, so we are looking for some subsheaf of the sheaf

$$
\mathcal{E} x t^{1}\left(\overline{\mathcal{M}}_{X_{0}(B, \mathscr{P}, s)}^{\mathrm{gp}}, \mathcal{O}_{X_{0}(B, \mathscr{P}, s)}^{\times}\right) .
$$

Here the superscript gp denotes the Grothendieck group of the monoid. Any extension of $\overline{\mathcal{M}}_{X_{0}(B, \mathscr{P}, s)}^{\mathrm{gp}}$ by $\mathcal{O}_{X_{0}(B, \mathscr{P}, s)}^{\times}$gives rise to a sheaf of groups $\mathcal{M}_{X_{0}(B, \mathscr{P}, s)}^{\mathrm{gp}}$ surjecting onto $\overline{\mathcal{M}}_{X_{0}(B, \mathscr{P}, s)}^{\mathrm{gp}}$, and the inverse image of $\overline{\mathcal{M}}_{X_{0}(B, \mathscr{P}, s)} \subseteq \overline{\mathcal{M}}_{X_{0}(B, \mathscr{P}, s)}^{\mathrm{gp}}$ is a sheaf of monoids $\mathcal{M}_{X_{0}(B, \mathscr{P}, s)}$. Of course, one also needs a map $\alpha: \mathcal{M}_{X_{0}(B, \mathscr{P}, s)} \rightarrow$ $\mathcal{O}_{X_{0}(B, \mathscr{P}, s)}$, and this complicates things a bit. To make a long story short, we can identify a subsheaf of extensions which yield genuine log structures. A section of this subsheaf determines a log structure on $X_{0}(B, \mathscr{P}, s)$ with the correct ghost sheaf. However, this is not precisely what we want. What we really want is a log structure on $X_{0}(B, \mathscr{P}, s)$ along with a $\log$ morphism $X_{0}(B, \mathscr{P}, s)^{\dagger} \rightarrow 0^{\dagger}$ which is $\log$ smooth. (We will address the question of the bad set $Z \subseteq \mathcal{X}_{0}$ shortly.) We call such a structure a $\log$ smooth structure on $X_{0}(B, \mathscr{P}, s)$. It turns out these structures are given by certain sections of $\mathcal{E} x t^{1}\left(\overline{\mathcal{M}}_{X_{0}(B, \mathscr{P}, s)}^{\mathrm{gp}} / \bar{\rho}, \mathcal{O}_{X}^{\times}\right)$, where $\bar{\rho}$ is the canonical section of $\overline{\mathcal{M}}_{X_{0}(B, \mathscr{P}, s)}$ whose germ at $\overline{\mathcal{M}}_{X_{0}(B, \mathscr{P}, s), \eta}=\mathbb{N}$ is 1 for $\eta$ a generic point of an irreducible component of $X_{0}(B, \mathscr{P}, s)$. So in fact, we can identify a subsheaf of $\mathcal{E} x t^{1}\left(\overline{\mathcal{M}}_{X_{0}(B, \mathscr{P}, s)}^{\mathrm{gp}} / \bar{\rho}, \mathcal{O}_{X}^{\times}\right)$, which we call $\mathcal{L S}_{X_{0}(B, \mathscr{P}, s)}$, whose sections determine a $\log$ structure on $X_{0}(B, \mathscr{P}, s)$ and a $\log$ smooth morphism $X_{0}(B, \mathscr{P}, s)^{\dagger} \rightarrow 0^{\dagger}$, i.e. a $\log$ smooth structure.

The technical heart of [22 is an explicit calculation of the sheaf $\mathcal{L S}_{X_{0}(B, \mathscr{P}, s)}$. This is carried out locally in $\mathbf{2 2}$, Theorem 3.22, where the sheaf is calculated on the (étale) open subsets $V(\sigma)$ of $X_{0}(B, \mathscr{P}, s)$, and globally in [22], Theorem 3.24. I will not state the precise results, but go into detail in a special case, which illustrates the most important features of the theory. 
Example 8.5. Suppose $X_{0}(B, \mathscr{P}, s)$ is normal crossings, i.e. every cell of $\mathscr{P}$ is affine isomorphic to a standard simplex. Then we have the local $\mathcal{T}^{1}$ sheaf,

$$
\mathcal{T}^{1}=\mathcal{E} x t_{X_{0}(B, \mathscr{P}, s)}^{1}\left(\Omega_{X_{0}(B, \mathscr{P}, s) / k}^{1}, \mathcal{O}_{X_{0}(B, \mathscr{P}, s)}\right) .
$$

This is a line bundle on $S=\operatorname{Sing}\left(X_{0}(B, \mathscr{P}, s)\right)$. Then one can show $\mathcal{L S}_{X_{0}(B, \mathscr{P}, s)}$ is the $\mathcal{O}_{S}^{\times}$-torsor associated to $\mathcal{T}^{1}$.

This brings us back to Friedman's condition of $d$-semistability [10. A variety with normal crossings is $d$-semistable if $\mathcal{T}^{1} \cong \mathcal{O}_{S}$. Thus we recover Kawamata and Namikawa's result $\left[36\right.$ showing that $X_{0}(B, \mathscr{P}, s)$ carries a normal crossings $\log$ structure over $0^{\dagger}$ if and only if $X_{0}(B, \mathscr{P}, s)$ is $d$-semistable. This is because, of course, the $\mathcal{O}_{S}^{\times}$-torsor associated to $\mathcal{T}^{1}$ has a section if and only if $\mathcal{T}^{1} \cong \mathcal{O}_{S}$.

Now Theorem 3.24 of $\left[22\right.$ tells us that in general $\mathcal{L S}_{X_{0}(B, \mathscr{P}, s)}$ is not a trivial $\mathcal{O}_{S}^{\times}$-torsor. The sheaf depends continuously on $s$, but discretely on monodromy of the singularities of $B$.

Let's explain the latter point explicitly if $\operatorname{dim} B=2$. The irreducible components of $S$ are in one-to-one correspondence with one-dimensional cells of $\mathscr{P}$. If $\omega \in \mathscr{P}$ is such an edge, suppose it contains one singularity of $B$ such that $\Lambda$ has monodromy $\left(\begin{array}{ll}1 & n \\ 0 & 1\end{array}\right)$ in a suitable basis around a loop around the singularity. Then $\mathcal{T}^{1}$ restricted to the one-dimensional stratum $X_{\omega} \cong \mathbb{P}^{1}$ of $X_{0}(B, \mathscr{P}, s)$ is $\mathcal{O}_{\mathbb{P}^{1}}(n)$.

To make this statement completely accurate, one needs to define $n$ so that it is independent of the choice of basis and loop. To do this, one chooses a loop which is counterclockwise with respect to the orientation determined by the chosen basis of $\Lambda_{b}=\mathcal{T}_{B, b}$, where $b \in B$ is the base-point of the loop.

If all the $n$ 's appearing are positive, then for some choices of gluing data $s$, we may hope to have a section $t$ of $\mathcal{T}^{1}$ which vanishes only at a finite set of points $Z$. If $Z$ does not contain a toric stratum (i.e. a triple point) then we obtain a log structure on $X_{0}(B, \mathscr{P}, s) \backslash Z$ of the desired sort, hence a log structure on $X_{0}(B, \mathscr{P}, s)(\log$ smooth off of $Z$ ) by push-forward. We then have

Proposition 8.6. In the situation of this example, with $\operatorname{dim} B=2$ and

$$
t \in \Gamma\left(X_{0}(B, \mathscr{P}, s), \mathcal{T}^{1}\right)
$$

a section vanishing on a finite set $Z$ not containing a triple point, there exists a smoothing $\mathcal{X} \rightarrow D$ of $X_{0}(B, \mathscr{P}, s)$ such that the singular locus of $\mathcal{X}$ is $Z \subseteq \mathcal{X}_{0}=$ $X_{0}(B, \mathscr{P}, s)$, and the induced log morphism $\mathcal{X}_{0}^{\dagger} \rightarrow 0^{\dagger}$ coincides with $X_{0}(B, \mathscr{P}, s)^{\dagger} \rightarrow$ $0^{\dagger}$ determined by $t$.

The proof of this is a rather simple application of Friedman's or Kawamata and Namikawa's results. To apply these results, however, we need to deal with the singular set $Z$. This is done by normalizing $X_{0}(B, \mathscr{P}, s)$, choosing to blow up one point in the inverse image of each point of $Z$, and then regluing along the proper transform of the conductor locus. This produces a $d$-semistable variety, in the language of Friedman, or a log smooth scheme, which can then be smoothed. (Such an approach seems difficult in higher dimensions.)

On the other hand, if $n<0$ for some singular point of $B$, we run into problems, and there is in fact no smoothing of $X_{0}(B, \mathscr{P}, s)$. This should not be surprising for the following reason. If $n=-1$, it turns out we would have to compactify the torus fibration $X\left(B_{0}\right)$ by adding a strange sort of $I_{1}$ fibre over such a singular point. An $I_{1}$ fibre is an immersed sphere, and the intersection multiplicity of the 
two sheets at the singular point of the fibre is +1 for an ordinary $I_{1}$ fibre. However, when the monodromy is given by $n=-1$, the intersection multiplicity is -1 . This does not occur for a special Lagrangian $T^{2}$-fibration, so it is not surprising we can't construct a smoothing in this case.

If $\operatorname{dim} B=2$ and $n>0$ for all singularities on $B$, then we say $B$ is positive. One can generalize this notion of positive to higher dimensional $B$ with polyhedral decompositions, see 22, Definition 1.54. Positivity of $B$ is a necessary condition for $X_{0}(B, \mathscr{P}, s)$ to appear as the central fibre of a toric degeneration. All the examples of $\S 4$ are positive; this in fact follows from the convexity of reflexive polytopes, and the positivity condition can be viewed as a type of convexity statement.

Passing back to the general case now, with no restriction on the dimension of $B$ or the shape of the cells of $\mathscr{P}$, it follows from [22. Theorem 3.24, that $\mathcal{L S}_{X_{0}(B, \mathscr{P}, s)}$ is a subsheaf of sets of a coherent sheaf we call $\mathcal{L S}_{\text {pre, } X_{0}(B, \mathscr{P}, s)}^{+}$. This sheaf is a direct sum $\bigoplus_{\substack{\omega \in \mathscr{P} \\ \operatorname{dim} \omega=1}} \mathcal{N}_{\omega}$, where $\mathcal{N}_{\omega}$ is a line bundle on the toric stratum of $X_{0}(B, \mathscr{P}, s)$ corresponding to $\omega$. Furthermore, as in the two-dimensional normal crossings case, $\mathcal{N}_{\omega}$ is a semi-ample line bundle if $B$ is positive.

A section $t \in \Gamma\left(X_{0}(B, \mathscr{P}, s), \mathcal{L S}_{\text {pre }, X_{0}(B, \mathscr{P}, s)}^{+}\right)$which is a section of $\mathcal{L S}_{X_{0}(B, \mathscr{P}, s)}$ outside of the zero set $Z$ of $t$ determines a $\log$ smooth structure on $X_{0}(B, \mathscr{P}, s) \backslash Z$. In particular, if $Z$ does not contain any toric stratum, we are in good shape. We then obtain a $\log$ morphism $X_{0}(B, \mathscr{P}, s)^{\dagger} \rightarrow 0^{\dagger}$ which is $\log$ smooth away from $Z$. We call such a structure a log Calabi-Yau space.

Let's review: given data

- $s$ open gluing data;

- $t \in \Gamma\left(X_{0}(B, \mathscr{P}, s), \mathcal{L S}_{\mathrm{pre}, X_{0}(B, \mathscr{P}, s)}^{+}\right)$, with $t$ a section of $\mathcal{L S}_{X_{0}(B, \mathscr{P}, s)}$ over $X_{0}(B, \mathscr{P}, s) \backslash Z$ for some set $Z$ which does not contain any toric stratum of $X_{0}(B, \mathscr{P}, s)$;

we obtain $X_{0}(B, \mathscr{P}, s)^{\dagger} \rightarrow 0^{\dagger}$.

Conversely, we show in 22 that if $\mathcal{X} \rightarrow D$ is a toric degeneration, then $\mathcal{X}_{0}^{\dagger} \rightarrow 0^{\dagger}$ is obtained in this way from the dual intersection complex $(B, \mathscr{P})$ from some choice of data $s$ and $t$. To complete this picture, it remains to answer

QUESTION 8.7 (The reconstruction problem, Version III). Suppose $(B, \mathscr{P})$ is positive.

(1) What are the possible choices of $s$ and $t$ which yield log Calabi-Yau spaces?

(2) Given $X_{0}(B, \mathscr{P}, s)^{\dagger} \rightarrow 0^{\dagger}$, when does it arise as the central fibre of a toric degeneration $\mathcal{X} \rightarrow D$ ?

As we have sketched it, this question is now the refined version of our basic reconstruction problem Question 7.11.

The choice of the data $s$ and $t$ determine the moduli of log Calabi-Yau spaces arising from a given dual intersection complex. So far we haven't even made the claim that this moduli space is non-empty, and for general choice of $(B, \mathscr{P})$, I do not know if this is the case or not, though it is non-empty if $\operatorname{dim} B=2$ or 3 . However, one would like a more explicit description of this moduli space in any event. In general the moduli space is a scheme, but it may be singular (an example is given in [22, Example 4.28). Some additional hypotheses are necessary to solve this problem. To motivate the necessary hypothesis, let's go back to $\S 1$, where 
we introduced the notion of simplicity. We saw that the basic topology of mirror symmetry works only when the fibration is simple. So maybe we should expect the current construction to work better when we have simplicity.

There is one technical problem with this: the definition of simplicity assumes the existence of a torus fibration $f: X \rightarrow B$. Instead, we want to define simplicity entirely in terms of $B$ itself. Unfortunately, the solution to this is rather technical, and produces a definition which is very difficult to absorb (Definition 1.60 of 22]). Let us just say here that if $B$ is simple in this new sense and $X\left(B_{0}\right) \rightarrow B_{0}$ was compactified in a sensible manner to a topological torus fibration $f: X(B) \rightarrow B$, then $f$ would be simple in the sense of $\S 1$, provided that $\operatorname{dim} B \leq 3$. In higher dimensions, this new simplicity does not necessarily imply the old simplicity; see the forthcoming Ph.D. thesis of Helge Ruddat. This arises in situations where orbifold singularities arise in $X(B)$; as is well-known, such singularities cannot be avoided in higher dimension.

Once we accept this definition, life simplifies a great deal. Extraordinarily, the a priori very complicated moduli space of log Calabi-Yau spaces with a given dual intersection complex has a very simple description when $B$ is simple! One very difficult main result of $\mathbf{2 2}$, (Theorem 5.4) is

Theorem 8.8. Given $(B, \mathscr{P})$ positive and simple, the set of log Calabi-Yau spaces with dual intersection complex $(B, \mathscr{P})$, modulo isomorphism preserving $B$, is $H^{1}\left(B, i_{*} \Lambda \otimes k^{\times}\right)$. An isomorphism is said to preserve $B$ if it induces the identity on the dual intersection complex.

So the moduli space is an algebraic torus (or a disjoint union of algebraic tori) of dimension equal to $\operatorname{dim}_{k} H^{1}\left(B, i_{*} \Lambda \otimes k\right)$.

Note that this is the expected dimension predicted by the SYZ conjecture. Indeed, if a smoothing of $X_{0}(B, \mathscr{P}, s)^{\dagger}$ exists and it was a topological compactification $X(B)$ of $X_{0}(B)$, with a simple torus fibration $f: X(B) \rightarrow B$ extending $f_{0}: X\left(B_{0}\right) \rightarrow B_{0}$, then $R^{n-1} f_{0 *} \mathbb{R} \cong \Lambda_{\mathbb{R}}$, so by simplicity, $R^{n-1} f_{*} \mathbb{R} \cong i_{*} \Lambda_{\mathbb{R}}$. The discussion of $\S 1$ suggests that $\operatorname{dim} H^{1}\left(B, R^{n-1} f_{*} \mathbb{R}\right)$ is $h^{1, n-1}$ of the smoothing, which is of course the dimension of the complex moduli space of the smoothing.

This argument can be made rigorous by introducing log differentials.

Definition 8.9. Let $\pi: X^{\dagger} \rightarrow S^{\dagger}$ be a morphism of logarithmic spaces. A log derivation on $X^{\dagger}$ over $S^{\dagger}$ with values in an $\mathcal{O}_{X}$-module $\mathcal{E}$ is a pair (D, Dlog), where D : $\mathcal{O}_{X} \rightarrow \mathcal{E}$ is an ordinary derivation of $X / S$ and Dlog: $\mathcal{M}_{X}^{\mathrm{gp}} \rightarrow \mathcal{E}$ is a homomorphism of abelian sheaves with $\operatorname{Dlog} \circ \pi^{\#}=0$; these fulfill the following compatibility condition

$$
\mathrm{D}\left(\alpha_{X}(m)\right)=\alpha_{X}(m) \cdot \operatorname{Dlog}(m),
$$

for all $m \in \mathcal{M}_{X}$.

We denote by $\Theta_{X^{\dagger} / S^{\dagger}}$ the sheaf of $\log$ derivations of $X^{\dagger}$ over $S^{\dagger}$ with values in $\mathcal{O}_{X}$. We set $\Omega_{X^{\dagger} / S^{\dagger}}^{1}=\operatorname{Hom}_{\mathcal{O}_{X}}\left(\Theta_{X^{\dagger} / S^{\dagger}}, \mathcal{O}_{X}\right)$.

This generalizes the more familiar notion of differentials with logarithmic poles along a normal crossings divisor. If $Y \subseteq X$ is a normal crossings divisor inducing a $\log$ structure on $X$, then $\Omega_{X^{\dagger} / k}^{1}$ is the sheaf of differentials with logarithmic poles along $Y$, and $\Omega_{Y^{\dagger} / k}^{1}$ is the restriction of this sheaf to $Y$. In general, $\Omega_{X^{\dagger} / S^{\dagger}}^{1}$ is locally free if $\pi$ is $\log$ smooth. As a result, one can do deformation theory in the log 
category for $\log$ smooth morphisms (see [34]). This is one of the principal reasons for introducing log geometry into our picture.

If $X_{0}(B, \mathscr{P}, s)^{\dagger} \rightarrow 0^{\dagger}$ is a $\log$ Calabi-Yau space, then the morphism is log smooth off of $Z$. Define

$$
\begin{array}{ll}
\Theta_{X_{0}(B, \mathscr{P}, s)}^{p} & :=j_{*} \bigwedge^{p} \Theta_{\left(X_{0}(B, \mathscr{P}, s)^{\dagger} \backslash Z\right) / 0^{\dagger}} \\
\Omega_{X_{0}(B, \mathscr{P}, s)}^{p} & :=j_{*} \bigwedge^{p} \Omega_{\left(X_{0}(B, \mathscr{P}, s)^{\dagger} \backslash Z\right) / 0^{\dagger}}^{1}
\end{array}
$$

where $j: X_{0}(B, \mathscr{P}, s) \backslash Z \rightarrow X_{0}(B, \mathscr{P}, s)$ is the inclusion.

Then one has

Theorem 8.10. Suppose $(B, \mathscr{P})$ is positive and simple, and suppose we are given a log Calabi-Yau space $X_{0}(B, \mathscr{P}, s)^{\dagger} \rightarrow 0^{\dagger}$ which occurs as the central fibre of a toric degeneration $\mathcal{X} \rightarrow D$ whose general fibre $\mathcal{X}_{t}$ is non-singular. Then for $q=0,1, n-1$ and $n$ with $n=\operatorname{dim} B$, we have isomorphisms

$$
\begin{aligned}
& H^{p}\left(B, i_{*} \bigwedge^{q} \Lambda \otimes k\right) \cong H^{p}\left(X_{0}(B, \mathscr{P}, s), \Theta_{X_{0}(B, \mathscr{P}, s)}^{q}\right) \cong H^{p}\left(\mathcal{X}_{t}, \Theta_{\mathcal{X}_{t}}^{q}\right) \\
& H^{p}\left(B, i_{*} \bigwedge^{q} \check{\Lambda} \otimes k\right) \cong H^{p}\left(X_{0}(B, \mathscr{P}, s), \Omega_{X_{0}(B, \mathscr{P}, s)}^{q}\right) \cong H^{p}\left(\mathcal{X}_{t}, \Omega_{\mathcal{X}_{t}}^{q}\right)
\end{aligned}
$$

where $\Theta_{\mathcal{X}_{t}}^{q}$ and $\Omega_{\mathcal{X}_{t}}^{q}$ are the ordinary sheaves of holomorphic poly-vector fields and holomorphic differentials on a smooth fibre $\mathcal{X}_{t}$.

The proof of this result, along with a number of other results, appears in 24. The result holds for all $q$ when additional hypotheses are assumed, essentially saying the mirror to $\mathcal{X}_{t}$ is non-singular. Note in particular, since $\Lambda$ and $\check{\Lambda}$ are interchanged under discrete Legendre transform, we get the interchange of ordinary Hodge numbers from this result. In the more general situation where the CalabiYaus arising are singular, one might speculate about the relationship between these groups, the actual Hodge numbers and stringy Hodge numbers. These issues are addressed in the forthcoming Ph.D. thesis of Helge Ruddat.

\section{The cone picture and the fan picture}

This section is purely philosophical. In most of our discussion in $\S \S 7$ and 8 , we focused on the dual intersection complex, and in particular, focused on the question of constructing a degeneration from its dual intersection complex. Since our primary goal was to solve the reconstruction problem Question [5.6, and as the dual intersection complex is related to the complex structure (Theorem 7.7) it seems natural to focus on the dual intersection complex. We will see in the next section that this intuition may not always be correct. So far, the intersection complex only seemed to arise when talking about mirror symmetry. However, mirror symmetry instructs us to view both sides of the picture on the same footing. When we construct a degenerate Calabi-Yau space from a dual intersection complex, we say we are in the fan picture, while if we construct a degenerate Calabi-Yau space from an intersection complex, we say we are in the cone picture.

More precisely, we have seen how given an integral affine manifold with singularities with toric polyhedral decomposition $(B, \mathscr{P})$, then an additional choice of open gluing data $s$ specifies a space $X_{0}(B, \mathscr{P}, s)$, along with a sheaf of monoids $\overline{\mathcal{M}}_{X_{0}(B, \mathscr{P}, s)}$. Some additional data may specify a $\log$ structure on $X_{0}(B, \mathscr{P}, s)$ with this ghost sheaf. The irreducible components of $X_{0}(B, \mathscr{P}, s)$ are defined using 
fans, given by the fan structure of $\mathscr{P}$ at each vertex of $\mathscr{P}$. This is why we call this side the fan picture.

On the other hand, given $(B, \mathscr{P})$ we can also construct a projective scheme $\check{X}_{0}(B, \mathscr{P}, \breve{s})$ given suitable gluing data $\check{s}$. The irreducible components of this scheme are in one-to-one correspondence with the maximal cells of $\mathscr{P}$; given such a maximal cell $\sigma$, viewing it as a lattice polytope in $\mathbb{R}^{n}$ determines a projective toric variety, and $\breve{X}_{0}(B, \mathscr{P}, \check{s})$ is obtained by gluing together these projective toric varieties using the data $\check{s}$. This is not quite the same data as occurred in the fan picture, because we also need to glue the line bundles, and this is additional data. The reason for calling this side the cone picture is that each irreducible component can be described as follows. Given $\sigma \subseteq M_{\mathbb{R}}$, let $P_{\sigma}=C(\sigma) \cap(M \oplus \mathbb{Z})$. Then the corresponding projective toric variety is Proj $\mathbb{C}\left[P_{\sigma}\right]$, where $\mathbb{C}\left[P_{\sigma}\right]$ is graded using the projection of $P_{\sigma}$ onto $\mathbb{Z}$. Hence the irreducible components and strata arise from cones over elements of $\mathscr{P}$.

We summarize the duality between the cone and fan pictures:

\begin{tabular}{|l|l|l|}
\hline & Fan picture & Cone picture \\
\hline Gluing data yields & $X_{0}(B, \mathscr{P}, s), \overline{\mathcal{M}}_{X_{0}(B, \mathscr{P}, s)}$ & $\bar{X}_{0}(B, \mathscr{P}, \check{s})$, ample line bundle \\
\hline$\sigma \in \mathscr{P}, \operatorname{dim} \sigma=p$ & $\begin{array}{l}\text { An } n-p \text {-dimensional stratum } \\
\text { of } X_{0}(B, \mathscr{P}, s)\end{array}$ & $\begin{array}{l}\text { A p-dimensional stratum } \\
\text { of } \check{X}_{0}(B, \mathscr{P}, \check{s})\end{array}$ \\
\hline $\begin{array}{l}\text { conulti-valued } \\
\text { convex PL function }\end{array}$ & $\begin{array}{l}\text { An ample line bundle } \\
\text { on } X_{0}(B, \mathscr{P}, s)\end{array}$ & $\begin{array}{l}\text { A sheaf of monoids } \\
\overline{\mathcal{M}}_{\check{X}_{0}(B, \mathscr{P}, \check{s})}\end{array}$ \\
\hline$H^{p}\left(B, i_{*} \bigwedge^{q} \Lambda \otimes k\right)$ & $H^{p}\left(X_{0}(B, \mathscr{P}, s), \Theta_{X_{0}(B, \mathscr{P}, s)}^{q}\right)$ & $H^{p}\left(X_{0}(B, \mathscr{P}, \check{s}), \Omega_{\tilde{X}_{0}(B, \mathscr{P}, \check{s})}^{q}\right)$ \\
\hline
\end{tabular}

(Some restrictions may apply to gluing data on both sides in order for $\varphi$ to yield the desired data.)

In particular, mirror symmetry interchanges discrete information about the log structure (i.e. $\overline{\mathcal{M}}_{X_{0}(B, \mathscr{P}, s)}$ ) and discrete information about the polarization (i.e the class of the line bundle on each irreducible component).

\section{Tropical curves}

So far we have seen only the most elementary aspects of mirror symmetry emerge from this algebro-geometric version of SYZ, e.g. the interchange of Hodge numbers. However, the real interest in this approach lies in hints that it will provide a natural explanation for rational curve counting in mirror symmetry. If we follow the philosophy of the previous section, we need to identify structures on affine manifolds with singularities which in one of the two pictures has to do with rational curves and in the other picture has to do with periods. I believe the correct structure to study is that of tropical curves on affine manifolds with singularities $B$. See [46], 50] for an introduction to tropical curves in $\mathbb{R}^{n}$. Here, we can take $B$ to be tropical, rather than integral; hence the name.

Definition 10.1. Let $B$ be a tropical affine manifold with singularities with discriminant locus $\Delta$. Let $G$ be a weighted, connected finite graph, with its set of vertices and edges denoted by $G^{[0]}$ and $G^{[1]}$ respectively, with weight function $w_{G}: G^{[1]} \rightarrow \mathbb{N} \backslash\{0\}$. A parametrized tropical curve in $B$ is a continuous map $h: G \rightarrow B$ satisfying the following conditions:

(1) For every edge $E \subseteq G,\left.h\right|_{\operatorname{Int}(E)}$ is an embedding, $h^{-1}\left(B_{0}\right)$ is dense in $\operatorname{Int}(E)$, and there is a section $u \in \Gamma\left(\operatorname{Int}(E), h^{*}\left(i_{*} \Lambda\right)\right)$ which is tangent to $h(\operatorname{Int}(E))$ at every point of $h(\operatorname{Int}(E)) \cap B_{0}$. We choose this section to be primitive, i.e. not an integral multiple of another section of $h^{*}\left(i_{*} \Lambda\right)$. 
(2) For every vertex $v \in G^{[0]}$, let $E_{1}, \ldots, E_{m} \in G^{[1]}$ be the edges adjacent to $v$. Let $u_{i}$ be the section of $\left.h^{*}\left(i_{*} \Lambda\right)\right|_{\operatorname{Int}\left(E_{i}\right)}$ promised by (1), chosen to point away from $v$. This defines germs $u_{i} \in h^{*}\left(i_{*} \Lambda\right)_{v}=\left(i_{*} \Lambda\right)_{h(v)}$.

(a) If $h(v) \in B_{0}$, the following balancing condition holds in $\Lambda_{h(v)}$ :

$$
\sum_{j=1}^{m} w_{G}\left(E_{j}\right) u_{j}=0
$$

(b) If $h(v) \notin B_{0}$, then the following balancing condition is satisfied in $\left(i_{*} \Lambda\right)_{h(v)}$ :

$$
\sum_{j=1}^{m} w_{G}\left(E_{j}\right) u_{j}=0 \quad \bmod \left(i_{*} \check{\Lambda}\right) \frac{\perp}{h(v)} \cap\left(i_{*} \Lambda\right)_{h(v)} .
$$

The latter group is interpreted as follows. Let $b \in B_{0}$ be a point near $h(v)$, and identify, via parallel transport along a path between $h(v)$ and $b$, the groups $\left(i_{*} \Lambda\right)_{h(v)}$ and $\left(i_{*} \check{\Lambda}\right)_{h(v)}$ with local monodromy invariant subgroups of $\Lambda_{b}$ and $\check{\Lambda}_{b}$ respectively. Then $\left(i_{*} \check{\Lambda}\right)_{h(v)}^{\perp}$ is a subgroup of $\Lambda_{b}$, and the intersection makes sense. It is independent of the choice of $b$ and path.

So tropical curves behave away from the discriminant locus of $B$ much as the tropical curves of [46], [50] do, but they may have legs terminating on the discriminant locus. As we are interested in the case that $B$ is compact, we do not want legs which go off to $\infty$. I warn the reader, however, that this definition is provisional, and the behaviour in (2) (b) may not be exactly what we want.

Here we see a tropical elliptic curve, the solid dots being points of the discriminant locus. The legs terminating at these points must be in a monodromy invariant direction.

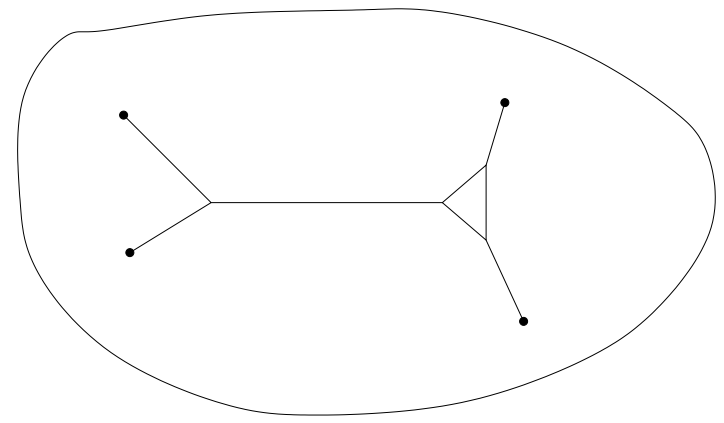

Now let us connect this to the question of counting curves. In the situation of a degeneration, $\varphi: \mathcal{X} \rightarrow D$, it is natural to consider families of maps of curves:

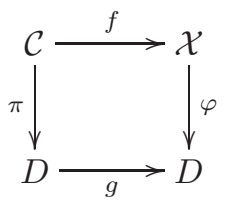

Here $g$ may be a ramified covering, and $\pi$ is a flat morphism with reduced onedimensional fibres. In the case of interest, $\left.f\right|_{\mathcal{C}_{t}}: \mathcal{C}_{t} \rightarrow \mathcal{X}_{t}$ should be a stable map of curves for $t \neq 0$. Let us assume that $\mathcal{C}_{t}$ is a non-singular curve for $t \neq 0$. In 
the logarithmic context, it is then natural to put the log structure induced by the divisor $\mathcal{C}_{0} \subseteq \mathcal{C}$ on $\mathcal{C}$, and so get a diagram

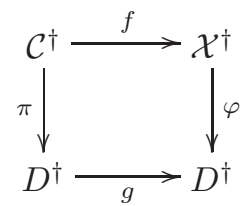

of log morphisms. Restricting to the central fibre, we obtain a diagram

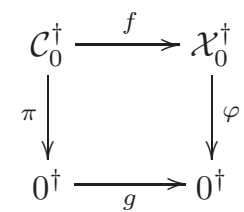

This suggests that we should build up a theory of stable log maps and log GromovWitten invariants. This theory should generalize the theories developed by $\mathrm{Li}$ and Ruan 42 and Jun Li 41. I will say little about this here, as this rapidly gets quite technical. There is work in progress of Siebert on this subject. This point of view has already been used in 48 for counting curves in toric varieties, so some more hints of this approach can be found there. Instead, I wish to sketch how such a diagram yields a tropical curve.

To do so, consider a situation where $\pi$ is normal crossings, and the induced map $\mathcal{C}_{0}^{\dagger} \rightarrow \mathcal{X}_{0}^{\dagger}$ has no infinitesimal log automorphisms over $0^{\dagger}$. (This is the log equivalent of the notion of stable map). Let $(B, \mathscr{P})$ be the dual intersection complex of the log Calabi-Yau space $\mathcal{X}_{0}^{\dagger}$. We can define the dual intersection graph of $f: \mathcal{C}_{0}^{\dagger} \rightarrow \mathcal{X}^{\dagger}$, which will be a parameterized tropical curve on $B$. I will only do the case here when the image of $f$ is disjoint from the set $Z \subseteq \mathcal{X}$ of Definition [7.1, (4); otherwise there are some technicalities to worry about.

First we build $G$. Let $C_{1}, \ldots, C_{m}$ be the irreducible components of $\mathcal{C}_{0}$. Assume these components are normal for ease of describing this construction. Set $G^{[0]}=$ $\left\{v_{1}, \ldots, v_{m}\right\}$. On the other hand, $G^{[1]}$ will contain an edge $\overline{v_{i} v_{j}}$ joining $v_{i}$ and $v_{j}$ whenever $C_{i} \cap C_{j} \neq \emptyset$.

To define $h: G \rightarrow B$, we first describe the image of each vertex. Let $X_{\sigma_{i}}$ be the minimal stratum of $\mathcal{X}_{0}$ containing $f\left(C_{i}\right)$, where $\sigma_{i} \in \mathscr{P}$. Let $\eta_{i}$ be the generic point of $C_{i}, \xi_{i}=f\left(\eta_{i}\right)$. Then we have an induced map $f^{\#}: \mathcal{M}_{\mathcal{X}_{0}, \xi_{i}} \rightarrow \mathcal{M}_{\mathcal{C}_{0}, \eta_{i}}$, as $f$ is a $\log$ morphism. This induces a diagram on stalks of ghost sheaves

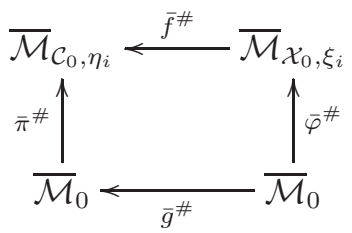

Now $\overline{\mathcal{M}}_{0}=\mathbb{N}$ (see Example 8.2, (4)) and $\overline{\mathcal{M}}_{\mathcal{C}_{0}, \eta_{i}}=\mathbb{N}$ since $\pi$ is normal crossings. On the other hand, $\bar{\pi}^{\#}$ is the identity and if $g$ is a branched cover of degree $d$, then $g^{\#}$ is multiplication by $d$. By Exercise 8.3,

$$
\overline{\mathcal{M}}_{\mathcal{X}_{0}, \xi_{i}}=\operatorname{Hom}_{\text {monoid }}\left(C\left(\sigma_{i}\right) \cap(M \oplus \mathbb{Z}), \mathbb{N}\right) .
$$


But

$$
\operatorname{Hom}_{\text {monoid }}\left(\operatorname{Hom}_{\text {monoid }}\left(C\left(\sigma_{i}\right) \cap(M \oplus \mathbb{Z}), \mathbb{N}\right), \mathbb{N}\right)=C\left(\sigma_{i}\right) \cap(M \oplus \mathbb{Z}),
$$

so $\bar{f}^{\#}$ is determined by an element $(m, r)$ of $C\left(\sigma_{i}\right) \cap(M \oplus \mathbb{Z})$. Now

$$
\bar{f}^{\#}\left(\bar{\varphi}^{\#}(1)\right)=\bar{f}^{\#}(0,1)=\langle(m, r),(0,1)\rangle=r
$$

while

$$
\bar{\pi}^{\#}\left(\bar{g}^{\#}(1)\right)=\bar{\pi}^{\#}(d)=d .
$$

Thus $r=d$, and $m / d \in \sigma_{i}$. We define $h\left(v_{i}\right)=m / d$. This is a point of $\sigma_{i}$ which is contained in $B$.

If $C_{i} \cap C_{j} \neq \emptyset$, there is a minimal stratum $X_{\sigma_{i, j}}$ containing $C_{i} \cap C_{j}$. Of course $X_{\sigma_{i, j}} \subseteq X_{\sigma_{i}} \cap X_{\sigma_{j}}$. In particular, $\sigma_{i, j}$ contains $\sigma_{i}$ and $\sigma_{j}$. We take $h\left(\overline{v_{i} v_{j}}\right)$ to be the straight line joining $h\left(v_{i}\right)$ and $h\left(v_{j}\right)$ inside $\sigma_{i, j}$. Furthermore, if $\sigma_{i, j} \subseteq M_{\mathbb{R}}$ is embedded as a lattice polytope, let $m_{i j}$ be a primitive lattice element parallel to $m_{i}-m_{j}$, and we take $w_{G}\left(\overline{v_{i} v_{j}}\right)$ to be defined by the equation

$$
w_{G}\left(\overline{v_{i} v_{j}}\right) m_{i j}=\#\left(C_{i} \cap C_{j}\right)\left(m_{i}-m_{j}\right) .
$$

\section{Proposition 10.2. $h$ is a parametrized tropical curve.}

We do not give a proof here. The case where $X_{\sigma_{i}}$ is always an irreducible component of $\mathcal{X}_{0}$ is essentially covered in [48. Instead, we'll do another extremal case, which exhibits some interesting features of log geometry.

Example 10.3. Suppose a component $C_{1}$ of $\mathcal{C}_{0}$ and all components $C_{2}, \ldots, C_{t}$ intersecting $C_{1}$ are mapped by $f$ to a zero dimensional stratum $X_{\sigma}$ of $\mathcal{X}_{0}$. Without loss of generality we can assume

$$
\mathcal{X}_{0}=V(\sigma)=\operatorname{Spec} \mathbb{C}\left[C(\sigma)^{\vee} \cap(N \oplus \mathbb{Z})\right] /\left(z^{(0,1)}\right)
$$

as defined in $\S 8$. Thus $h$ maps $v_{1}, \ldots, v_{t}$ into points $m_{1} / d, \ldots, m_{t} / d \in \sigma$. Let us understand why the balancing condition holds at $m_{1} / d$. Let $U \subseteq \mathcal{C}_{0}$ be an open neighbourhood of $C_{1}$ which only intersects $C_{1}, \ldots, C_{t}$, so $h$ is constant on $U$ as an ordinary morphism (but not as a log morphism). Restrict the log structure on $\mathcal{C}_{0}$ to $U$. We have an exact sequence

$$
1 \longrightarrow \mathcal{O}_{U}^{\times} \longrightarrow \mathcal{M}_{U}^{\mathrm{gp}} \stackrel{p}{\longrightarrow} \overline{\mathcal{M}}_{U}^{\mathrm{gp}} \longrightarrow 0 .
$$

Taking global sections, we get

$$
1 \longrightarrow \Gamma\left(U, \mathcal{O}_{U}^{\times}\right) \longrightarrow \Gamma\left(U, \mathcal{M}_{U}^{\mathrm{gp}}\right) \stackrel{p}{\longrightarrow} \Gamma\left(U, \overline{\mathcal{M}}_{U}^{\mathrm{gp}}\right)=\mathbb{Z}^{t} \stackrel{q}{\longrightarrow} \operatorname{Pic} U .
$$

A section $s \in \Gamma\left(U, \overline{\mathcal{M}}_{U}^{\mathrm{gp}}\right)$ defines an $\mathcal{O}_{U}^{\times}$-torsor $p^{-1}(s)$, whose class in the Picard group of $U$ is $q(s)$. It is an easy exercise in $\log$ geometry to show that if $s$ is the $i$ th standard basis vector for $\mathbb{Z}^{t}$, then $q(s)=\left.\mathcal{O}_{\mathcal{C}}\left(-C_{i}\right)\right|_{U}$. Note $\left.\operatorname{deg} \mathcal{O}_{\mathcal{C}}\left(-C_{i}\right)\right|_{C_{1}}=$ $-\# C_{1} \cap C_{i}$ for $i=2, \ldots, t$ and $\left.\operatorname{deg} \mathcal{O}_{\mathcal{C}}\left(-C_{1}\right)\right|_{C_{1}}=\sum_{i=2}^{t} \# C_{1} \cap C_{i}$ as $C_{1} \cdot \mathcal{C}_{0}=0$ in $\mathcal{C}$.

Now observe that $f^{\#}$ acting on the sheaves of monoids induces a diagram

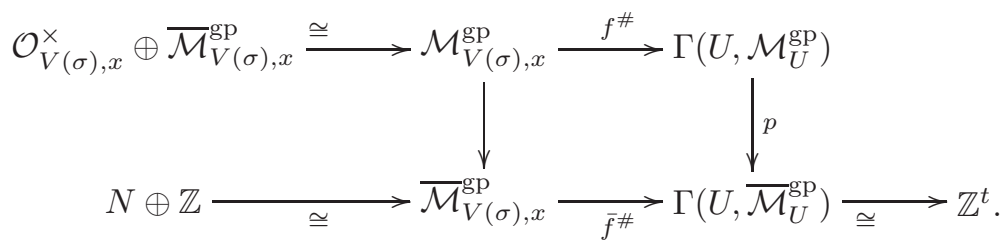


The map $\bar{f}^{\#}$, by construction, is given by

$$
(n, r) \in N \oplus \mathbb{Z} \mapsto\left(\left\langle(n, r),\left(m_{i}, d\right)\right\rangle\right)_{i=1, \ldots, t} .
$$

On the other hand, in order for $\bar{f}^{\#}$ to lift to $f^{\#}$, the $\mathcal{O}_{U}^{\times}$torseur $p^{-1}\left(\bar{f}^{\#}(n, r)\right)$ must have a section for every $(n, r) \in N \oplus \mathbb{Z}$, i.e. must be trivial in the Picard group. This implies

or

$$
\operatorname{deg} \bigotimes_{i=1}^{t}\left(\left.\mathcal{O}_{\mathcal{C}}\left(-C_{i}\right)\right|_{C_{1}}\right)^{\otimes\left\langle(n, r),\left(m_{i}, d\right)\right\rangle}=0
$$

$$
\sum_{i=2}^{t}\left(\# C_{1} \cap C_{i}\right)\left(\left\langle(n, r),\left(m_{i}, d\right)-\left(m_{1}, d\right)\right\rangle\right)=0
$$

for all $(n, r) \in N \oplus \mathbb{Z}$. But this is equivalent to

$$
\sum_{i=2}^{t}\left(\# C_{1} \cap C_{i}\right)\left(m_{i}-m_{1}\right)=0
$$

which is the balancing condition.

Following the logic of mirror symmetry, this suggests that tropical curves on the cone side should have to do with periods. It is only recently that an understanding of this has begun to emerge, and unfortunately, I do not have space or time to elaborate on this. Let me say that in 23, Siebert and I have given a solution to Question 8.7. (2), given some hypotheses on $X_{0}(B, \mathscr{P}, s)^{\dagger}$, which are implied by simplicity of $B$. In this solution, we construct explicit deformations of a log Calabi-Yau space, order by order. Formally, our construction looks somewhat similar to that of Kontsevich and Soibelman [39] for constructing non-Archimedean K3 surfaces from affine manifolds, and we apply a key lemma of [39. However, Kontsevich and Soibelman work on what we would call the fan side, while we work on the cone side. This may be surprising given that all of our discussions involving the strategy of building log Calabi-Yau spaces was done on the fan side. However, if we take the mirror philosophy seriously, and we want to see tropical curves appear in a description of a smoothing, we need to work on the cone side. It turns out to be extremely natural. In fact, all tropical rational curves play a role in our construction. Ultimately, all periods can be calculated in terms of the data involved in our construction, and in particular, there is a clear relationship between the period calculation and the existence of tropical rational curves on $B$. Once this is fully understood, this will finally give a firm understanding of a geometric explanation of mirror symmetry.

\section{References}

[1] P. Aspinwall, B. Greene and D. Morrison: Calabi-Yau moduli space, mirror manifolds and spacetime topology change in string theory, Nuclear Phys. B416 (1994), 414-480.

[2] V. Batyrev: Dual polyhedra and mirror symmetry for Calabi-Yau hypersurfaces in toric varieties. J. Algebraic Geom. 3 (1994), 493-535.

[3] V. Batyrev, and L. Borisov: On Calabi-Yau complete intersections in toric varieties, in Higher-dimensional complex varieties (Trento, 1994), 39-65, de Gruyter, Berlin, 1996.

[4] V. Batyrev, and M. Kreuzer: Integral cohomology and mirror symmetry for Calabi-Yau 3-folds, preprint, 2005, math.AG/0505432.

[5] O. Ben-Bassat, Mirror symmetry and generalized complex manifolds, preprint, 2004, math. AG/0405303. 
[6] A. Bertram, Another way to enumerate rational curves with torus actions, Invent. Math. 142 (2000), 487-512.

[7] R. Castaño-Bernard and D. Matessi, Lagrangian 3-torus fibration, preprint, 2006, arXiv:math/0611139.

[8] P. Candelas, X. de la Ossa, P. Green, and L. Parkes, A pair of Calabi-Yau manifolds as an exactly soluble superconformal theory, Nuclear Phys. B 359 (1991), 21-74.

[9] S.-Y. Cheng and S.-T. Yau, The real Monge-Ampère equation and affine flat structures, in Proceedings of the 1980 Beijing Symposium on Differential Geometry and Differential Equations, Vol. 1, 2, 3 (Beijing, 1980), 339-370, Science Press, Beijing, 1982.

[10] R. Friedman: Global smoothings of varieties with normal crossings, Ann. Math. 118, (1983) 75-114.

[11] K. Fukaya, Multivalued Morse theory, asymptotic analysis and mirror symmetry, in Graphs and patterns in mathematics and theoretical physics, 205-278, Proc. Sympos. Pure Math., 73, Amer. Math. Soc., Providence, RI, 2005.

[12] A. Gathmann, Relative Gromov-Witten invariants and the mirror formula, Math. Ann. 325 (2003), 393-412.

[13] A. Givental, Equivariant Gromov-Witten invariants, Internat. Math. Res. Notices 13, (1996), 613-663.

[14] E. Goldstein: A construction of new families of minimal Lagrangian submanifolds via torus actions, J. Differential Geom. 58 (2001), 233-261.

[15] M. Gross: Special Lagrangian Fibrations I: Topology, in: Integrable Systems and Algebraic Geometry, (M.-H. Saito, Y. Shimizu and K. Ueno eds.), World Scientific 1998, 156-193.

[16] M. Gross: Special Lagrangian Fibrations II: Geometry, in: Surveys in Differential Geometry, Somerville: MA, International Press 1999, 341-403.

[17] M. Gross: Topological Mirror Symmetry, Invent. Math. 144 (2001), 75-137.

[18] M. Gross: Examples of special Lagrangian fibrations, in Symplectic geometry and mirror symmetry (Seoul, 2000), 81-109, World Sci. Publishing, River Edge, NJ, 2001.

[19] M. Gross: Toric Degenerations and Batyrev-Borisov Duality, Math. Ann. 333, (2005) 645-688.

[20] M. Gross, and B. Siebert: Affine manifolds, log structures, and mirror symmetry, Turkish J. Math. 27 (2003), 33-60.

[21] M. Gross, and B. Siebert: Torus fibrations and toric degenerations, in preparation.

[22] M. Gross, and B. Siebert: Mirror symmetry via logarithmic degeneration data I, J. Diff. Geom. 72, (2006).

[23] M. Gross, and B. Siebert: From real affine geometry to complex geometry, preprint, (2007), arXiv:math/073822.

[24] M. Gross, and B. Siebert: Mirror symmetry via logarithmic degeneration data II, preprint, (2007), arXiv:0709.2290.

[25] M. Gross, and P.M.H. Wilson: Mirror symmetry via 3-tori for a class of Calabi-Yau threefolds, Math. Ann. 309 (1997), 505-531.

[26] M. Gross, and P.M.H. Wilson: Large complex structure limits of K3 surfaces, J. Differential Geom. 55 (2000), 475-546.

[27] M. Gualtieri, Generalized complex geometry, Oxford University DPhil thesis, math.DG/0401221.

[28] C. Haase, and I. Zharkov: Integral affine structures on spheres and torus fibrations of Calabi-Yau toric hypersurfaces I, preprint 2002, math.AG/0205321.

[29] C. Haase, and I. Zharkov: Integral affine structures on spheres III: complete intersections, preprint, math.AG/0504181.

[30] N. Hitchin: The Moduli Space of Special Lagrangian Submanifolds, Ann. Scuola Norm. Sup. Pisa Cl. Sci. (4) 25 (1997), 503-515.

[31] N. Hitchin: Generalized Calabi-Yau manifolds, Q. J. Math. 54 (2003), 281-308.

[32] L. Illusie: Logarithmic spaces (according to K. Kato), in Barsotti Symposium in Algebraic Geometry (Abano Terme 1991), 183-203, Perspect. Math. 15, Academic Press 1994.

[33] D. Joyce, Singularities of special Lagrangian fibrations and the $S Y Z$ conjecture, Comm. Anal. Geom. 11 (2003), 859-907.

[34] F. Kato: Log smooth deformation theory, Tohoku Math. J. 48 (1996), 317-354. 
[35] K. Kato: Logarithmic structures of Fontaine-Illusie, in: Algebraic analysis, geometry, and number theory (J.-I. Igusa et. al. eds.), 191-224, Johns Hopkins Univ. Press, Baltimore, 1989.

[36] Y. Kawamata, Y. Namikawa: Logarithmic deformations of normal crossing varieties and smooothing of degenerate Calabi-Yau varieties, Invent. Math. 118 (1994), 395-409.

[37] M. Kontsevich, Enumeration of rational curves via torus actions, in The moduli space of curves (Texel Island, 1994), 335-368, Progr. Math., 129, Birkhäuser Boston, Boston, MA, 1995.

[38] M. Kontsevich, and Y. Soibelman: Homological mirror symmetry and torus fibrations, in: Symplectic geometry and mirror symmetry (Seoul, 2000), 203-263, World Sci. Publishing, River Edge, NJ, 2001.

[39] M. Kontsevich, and Y. Soibelman: Affine structures and non-archimedean analytic spaces, preprint, math.AG/0406564.

[40] N.C. Leung: Mirror symmetry without corrections, preprint 2000, math.DG/0009235

[41] J. Li, Stable morphisms to singular schemes and relative stable morphisms, J. Differential Geom. 57 (2001), 509-578.

[42] A-M. Li, and Y. Ruan: Symplectic surgery and Gromov-Witten invariants of Calabi-Yau 3-folds, Invent. Math. 145 (2001), 151-218.

[43] B. Lian, K. Liu, S-T. Yau, Mirror principle. I, Asian J. Math. 1 (1997), 729-763.

[44] D. Matessi, Some families of special Lagrangian tori, Math. Ann. 325 (2003), 211-228.

[45] R. McLean, Deformations of calibrated submanifolds, Comm. Anal. Geom. 6 (1998), $705-747$.

[46] G. Mikhalkin, Enumerative tropical algebraic geometry in $\mathbb{R}^{2}$, J. Amer. Math. Soc. 18 (2005), 313-377.

[47] D. Morrison, Compactifications of moduli spaces inspired by mirror symmetry, in Journées de Géométrie Algébrique d'Orsay (Orsay, 1992), Astrisque 218 (1993), 243271.

[48] T. Nishinou, B. Siebert, Toric degenerations of toric varieties and tropical curves, preprint, math.AG/0409060, to appear in Duke Math. Journal.

[49] P. Petersen: Riemannian geometry, Graduate Texts in Mathematics, 171. SpringerVerlag, New York, 1998.

[50] J. Richter-Gebert, B. Sturmfels, and T. Theobald, First steps in tropical geometry, in Idempotent mathematics and mathematical physics, 289-317, Contemp. Math., 377, Amer. Math. Soc., Providence, RI, 2005.

[51] E. Rødland: The Pfaffian Calabi-Yau, its mirror, and their link to the Grassmannian $G(2,7)$, Compositio Math. 122, (2000) 135-149.

[52] W.-D. Ruan: Lagrangian torus fibration and mirror symmetry of Calabi-Yau hypersurface in toric variety, preprint 2000, math.DG/0007028.

[53] W.-D. Ruan: Lagrangian torus fibration of quintic Calabi-Yau hypersurfaces. II. Technical results on gradient flow construction, J. Symplectic Geom. 1 (2002), no. 3, 435-521.

[54] S. Schröer, B. Siebert: Irreducible degenerations of primary Kodaira surfaces, in Complex geometry (Göttingen, 2000), 193-222, Springer, Berlin, 2002.

[55] S. Schröer, B. Siebert: Toroidal crossings and logarithmic structures, preprint 2002, math.AG/0211088, to appear in Adv. Math.

[56] A. Strominger, S.-T. Yau, and E. Zaslow, Mirror Symmetry is T-duality, Nucl. Phys. B479, (1996) 243-259.

UCSD Mathematics, 9500 Gilman Drive, La Jolla, CA 92093-0112, USA

E-mail address: mgross@math.ucsd.edu 